\title{
Anabases
}

ANABASES Traditions et réceptions de l'Antiquité

13 | 2011

Varia

\section{Entretien : Maurice Sartre et le métier d'historien}

\section{Maurice Sartre}

\section{OpenEdition}

Journals

Édition électronique

URL : http://journals.openedition.org/anabases/1886

DOI : 10.4000/anabases. 1886

ISSN : 2256-9421

\section{Éditeur}

E.R.A.S.M.E.

\section{Édition imprimée}

Date de publication : 1 mars 2011

Pagination : 175-206

ISSN : 1774-4296

\section{Référence électronique}

Maurice Sartre, «Entretien : Maurice Sartre et le métier d'historien », Anabases [En ligne], 13 | 2011, mis en ligne le 01 mars 2014, consulté le 20 octobre 2019. URL : http://journals.openedition.org/ anabases/1886 ; DOI : 10.4000/anabases.1886

Ce document a été généré automatiquement le 20 octobre 2019.

(c) Anabases 


\title{
Entretien : Maurice Sartre et le métier d'historien
}

\author{
Maurice Sartre
}

\section{NOTE DE L'ÉDITEUR}

Nous proposons ici le texte à peine retouché d'un entretien avec Maurice Sartre qui s'est déroulé le 14 mai 2010, à l'Université de Toulouse II - Le Mirail, dans le cadre du séminaire annuel de l'équipe PLH-ERASME. Nous avons conservé pour l'essentiel la spontanéité et la tournure orale des échanges, et ajouté quelques notes pour aider les lecteurs. Nous remercions Nathaël Recoursé, doctorant, pour une première transcription de l'enregistrement intégral, et Maurice Sartre, à la fois pour sa révision et pour la générosité qu'il a manifestée lors de cette visite. L'article qui suit est comme une mise en œuvre des principes exposés et discutés au cours de ces échanges.

PASCAL PAYEN : La meilleure façon de rendre hommage à un historien, à ses travaux, à ses recherches, est certainement de le convier à en parler et de dialoguer avec lui sur son métier. Maurice Sartre a de longues années de pratique de ce métier et de débat sur l'évolution des sciences historiques et sur les rapports entre recherche et diffusion des connaissances. Ses analyses peuvent donc intéresser tous les historiens que nous sommes, jeunes et moins jeunes. Faire venir Maurice Sartre, que nous remercions d'avoir fait le voyage spécialement de Syrie pour cette occasion, était un projet que nous avions en tête depuis quelques années, parce que la diversité de ses activités d'historien et au service de l'histoire correspond bien à l'esprit que nous tentons d'impulser au sein de l'équipe ERASME, autour de l'Antiquité et de ses réceptions. Pour ouvrir la table ronde en songeant à la diversité des publics qui sont réunis, je voudrais tout d'abord présenter l'œuvre de Maurice Sartre, en essayant de dégager, livres à l'appui, ce qui constitue les lignes de force de son travail d'historien et de son activité.

Le premier registre dans lequel se situent ses travaux est celui de l'érudition. Le territoire de Maurice Sartre historien est la Syrie ancienne, le Levant antique, une très vaste région couverte par les États modernes du Liban, d'Israël, des territoires palestiniens, de la Syrie et de la Jordanie. Maurice Sartre a ainsi édité de nombreuses inscriptions depuis 1982, lorsqu'il publie, dans les Inscriptions grecques et latines de Syrie, le volume 13.1, consacré à 
la cité de Bostra', la capitale de la province romaine d'Arabie, dont il est le spécialiste, comme en témoigne la monographie qu'il lui consacre en $1985^{2}$. Dans sa carrière, ce sont plus de 4000 inscriptions qu'il a trouvées, accompagné de son épouse, historienne elle aussi, qu'il a éditées et commentées avec elle.

Le deuxième registre se situe à la croisée du domaine savant et des activités d'enseignement. Maurice Sartre est l'auteur de grandes synthèses concernant l'histoire du Proche-Orient à l'époque hellénistique et romaine. Le premier ouvrage rédigé en ce sens est L'Orient romain. Provinces et sociétés provinciales en Méditerranée orientale d'Auguste aux Sévères, 31 av. J.-C. - 235 ap. J.-C., publié en 1991³. Mais celui qui me séduit le plus dans cette vaste production est un autre livre de synthèse, dont il dit qu'il l'a écrit avant tout pour lui-même et non pas pour le public: D'Alexandre à Zénobie. Histoire du Levant antique, Iv siècle av. J.-C. - III siècle ap. J.-C., paru en $2001^{4}$.

Dans le même registre, on signalera encore les manuels, c'est-à-dire des livres qui permettent à des générations d'étudiants, mais aussi de collègues, de se former, en particulier ceux qui ont paru dans la collection $U$, chez Armand Colin : L'Asie mineure et I'Anatolie, d'Alexandre à Dioclétien ıv siècle av. J.-C. - II $^{e}$ siècle ap. J.-C. ${ }^{5}$ et L'Anatolie hellénistique, de l'Égée au Caucase ${ }^{6}$.

Une troisième facette de ce vaste travail est la diffusion du savoir historique, destinée à toucher des publics plus larges que ceux des milieux universitaires. Je voudrais signaler à ce sujet un livre passionnant: Histoires grecques, paru en 2006 et constitué de quarantetrois courts chapitres reposant sur une source, d'abord traduite, puis objet d'un commentaire historique ${ }^{7}$. Ce livre est une vraie leçon de méthode, et il est déjà traduit en anglais, aux États-Unis, avec des traductions en préparation en serbe, turc et grec. Je signale également, dans le même registre, un livre intitulé La Syrie antique ${ }^{8}$. Enfin, il faut souligner la place que prend Maurice Sartre dans la revue L'Histoire où il siège depuis maintenant quinze ans au comité de rédaction. De même, chacun connaît sa participation active au Monde des livres et son rôle dans les débuts des «Rendez-vous de I'histoire » à Blois.

Ces trois registres : l'érudition scientifique, l'écriture de larges synthèses, les formes variées de la diffusion du savoir, sont, reconnaissons-le, fort difficiles à concilier, ne serait-ce que pour des raisons de temps. J'ajoute que Maurice Sartre a été un enseignant, un universitaire, faisant des cours, participant à des jurys de concours, dirigeant des mémoires de toute sorte et de très nombreuses thèses.

C'est donc autour de ces travaux que nous allons échanger, enseignants-chercheurs, doctorants et public. II sera question de l'histoire et de l'archéologie du Proche-Orient, et des rapports entre Orient ancien et Orient moderne; nous discuterons aussi de l'engagement de l'historien dans les formes de diffusion du savoir et des dossiers qui semblent les plus prometteurs dans le domaine de I'histoire de l'Antiquité, y compris les questions de réception qui nous tiennent spécialement à cœur.

J'ouvre le débat par une première question. Depuis le début des années '60, l'étude de l'Antiquité n'est nullement restée à l'écart des grands renouvellements historiographiques qui ont touché l'ensemble des disciplines historiques, en particulier par la rencontre avec d'autres disciplines : je pense à la psychologie historique et à l'anthropologie - et je renvoie à l'œuvre de Jean-Pierre Vernant -, mais aussi avec la sociologie, du côté de Paul Veyne, dont le grand livre Le pain et le cirque est sous-titré Sociologie historique d'un pluralisme politique ${ }^{9}$. Le renouvellement s'est opéré aussi du côté des thématiques, par exemple avec I'histoire des rapports entre les sexes. Quelle appréciation d'ensemble portez-vous sur ce renouvellement du questionnement dans le champ historiographique depuis quarante ou cinquante ans? Ce contexte a-t-il exercé une influence sur vos propres recherches et, si c'est le cas, en quel sens?

Maurice Sartre : Ce n'est pas par esprit de contradiction, encore que l'historien se doit d'avoir l'esprit de contradiction, mais je vais répondre aux questions en sens inverse. D’abord, merci pour cette présentation, qui souligne l'une de mes plus profondes convictions: je crois effectivement que l'érudition est le fondement de 
tout, et il ne faut pas en avoir honte. Il n'y a pas d'historien-chercheur qui ne soit pas d'abord un érudit. Dans le fond, ce qui nous donne une légitimité pour parler de questions plus larges, c'est la maîtrise, aussi précise que possible, d'une connaissance spécialisée. Pour revenir aux questions : je crois que la situation de l'histoire dans les années ‘60-'70 (j’étais étudiant dans les années 1960, et ma carrière universitaire a commencé en 1969), était bien différente de celle d'aujourd'hui, sur un point au moins, celui de l'idéologie. Il existait alors au sein de l'école historique française - et l'Antiquité était partie prenante de cette école - des clans et des chapelles aux positions tranchées. Il y avait des positions idéologiques en apparence inconciliables, avec, d'un côté, ceux qui prônaient une histoire tournée vers la sociologie, l'anthropologie et plus largement les sciences sociales (il faudrait ajouter l'histoire économique, mais cela concernait moins l'Antiquité) - on peut rappeler les noms de Veyne et de Vernant, qui sont pour moi deux savants considérables. Ils ont été des initiateurs formidables et leurs erreurs éventuelles importent moins que le fait qu'ils aient obligé chacun à réfléchir de manière différente et à trouver, le cas échéant, de meilleures réponses aux questions que ces savants ont été les premiers à poser. Dans le renouvellement d'une discipline, ce qui est important, ce ne sont pas tant les réponses que les questions que l'on se pose. Face à ces gens-là, qui essayaient d'ouvrir sur la modernité, il y avait ce que j'appellerais le «clan des philologues », héritiers d'une formidable et indispensable érudition, mais qui préféraient se concentrer sur la forme du iota - je caricature, il va sans dire - plutôt que de problématiser. Il faut dire aussi, même si les recoupements sont infiniment complexes, que les positions idéologiques des individus, fortement marquées par les prises de position face à la guerre froide et lors des guerres coloniales, accentuaient encore l'opposition des hommes dans ce qui apparaissait, à mes yeux d'étudiant du moins, comme un nouvel avatar du combat des Anciens et des Modernes. Les haines pouvaient être inexpiables entre les clans, même lorsque, sur le fond, on apercevait de vraies convergences scientifiques.

Pour ma part, j'étais persuadé qu'il fallait à la fois maîtriser l'érudition et garder l'œil ouvert sur ce qui se passait ailleurs. Car la forme d'une lettre - pour reprendre mon allusion caricaturale d'il y a un instant - peut avoir beaucoup d'importance. Je ne dis pas ça tout à fait par hasard : vous lirez si vous en avez envie un chapitre des Histoires grecques où j'ai essayé d'expliquer comment l'analyse que l'on faisait alors de la nature même de l'impérialisme athénien dépendait beaucoup de la forme du sigma dans un décret attique, et tout autant, peut-être, de la manière dont on se positionnait face à l'idéologie anti-impérialiste des années ' $70^{10}$. La date du décret de Cléarque sur les poids et mesures d'Athènes, qui a fait couler tant d'encre alors, ne semble plus guère déchaîner les passions : mais il est vrai que la guerre du Vietnam est terminée depuis plus de 30 ans !

Je reste naturellement persuadé que l'érudition la plus stricte est indispensable ; mais en même temps je suis convaincu que tous ceux qui nous ouvrent les yeux et l'esprit vers d'autres horizons nous enrichissent. Je n'ai jamais appartenu à aucune chapelle, mais j'ai manifesté beaucoup d'intérêt pour ce que faisaient Vernant, Veyne, VidalNaquet, qui suscitaient jadis des oppositions, voire des haines inimaginables. Aujourd'hui personne ne songerait à remettre en cause sérieusement tout ce qu'ils ont apporté, même si certains s'en tiennent encore à l'écart. Ce qui ne veut pas dire que tout était ni exact ni facile. Car ce courant de la recherche avait ses travers, 
comme le goût pour le jargon. Il m'est arrivé, je le dis honnêtement, de lire du Paul Veyne sans comprendre un traitre mot de ce qu'il écrivait, pour des raisons de vocabulaire ou de style. On n'a pas cité le nom de Nicole Loraux, pour qui j'ai aussi une grande admiration, mais une admiration critique en même temps. Peu importe qu'elle se soit trompée sur certains points, qu'elle ait parfois mêlé des textes d'époques trop différentes, mais je lui en veux surtout d'avoir fait le choix d'un discours obscurci par l'usage d'une langue pour initiés : quiconque n'avait pas suivi son séminaire à l'EHEss pendant des années comprenait avec peine ce qu'elle écrivait. Il n'empêche qu'il valait la peine de percer le mur de l'incompréhension pour voir ce qu'il y avait derrière, qui était porteur d'avenir.

J'ai beaucoup tiré profit, même si je me garderai bien de me comparer à ceux que je considère comme des géants de notre discipline, de cette combinaison de l'érudition à l'état pur, d'une part, celle que m'a enseignée mon maitre Jean Pouilloux, celle que j'ai apprise à la lecture de Louis Robert, qui se situe dans le droit fil de l'érudition allemande du xIX siècle et, d'autre part, des apports de ceux qui ouvrent des portes, qui sollicitent notre curiosité. Avec le recul, on s'aperçoit que les vrais savants, ceux qui paraissaient au départ confinés dans l'érudition la plus stricte (je pense à Louis Robert), quand on les lit attentivement, sont aussi de formidables initiateurs et contribuent à leur manière à la marche des idées. Louis Robert n'a jamais dit qu'il faisait de la sociologie historique ; il n'a jamais employé le vocabulaire de Vernant, de Vidal-Naquet, mais, pour avoir connu ceux-ci et surtout le second, je peux dire qu'ils étaient des admirateurs réels de Louis Robert et fréquentaient son œuvre plus que quiconque. Car il y a déjà dans l'œuvre de Louis Robert une grande partie de ce que ces savants ont formalisé, finalisé, problématisé. Quel est le plus grand historien des sociétés grecques d'époque hellénistique et impériale ? Louis Robert, évidemment. On comprend qu'il est vain d'opposer les groupes les uns aux autres quand, de chaque côté, l'érudition est reine.

Aujourd'hui quelle est la situation? Je partirai d'un constat. Depuis une quinzaine d'années j'œuvre beaucoup dans la critique, puisque depuis 1996 je tiens l'essentiel des comptes rendus d'histoire ancienne dans Le Monde des Livres et dans L'Histoire. Ce qui m'inquiète, c'est que la disparition ou le silence de ces géants, puisque Paul Veyne a dit qu'après son livre sur Michel Foucault il n'écrirait plus rien ${ }^{11}$, nous laisse dans une sorte de désert. Depuis presque un an, je n'ai guère vu arriver de livres qui soient vraiment stimulants, innovants ${ }^{12}$. Personne n'a remplacé ces maîtres, et cela m'inquiète. Dans le fond, la « disparition des idéologies » fait qu'aujourd'hui l'histoire ancienne, j'ai le regret de le dire, paraît un peu à la traîne des autres périodes historiques.

Dans les années '60-'70, les historiens de l'Antiquité ont beaucoup innové, créant des pistes nouvelles qui ont suscité de l'intérêt dans les autres périodes - je pense notamment aux études de vocabulaire, avec Pierre Lévêque et l'équipe de Besançon, qui ont innové, publié beaucoup, même si certains travaux sont aujourd'hui périmés après avoir été des outils formidables. La série des colloques sur l'esclavage, par exemple, peut être aujourd'hui à bon droit critiquée, et c'est notre rôle, mais elle a été très utile! Or, ces études de vocabulaire ont été imitées par tout le monde, du Moyen Âge jusqu'à l'époque la plus contemporaine. Maintenant, nous sommes un peu à la traîne en histoire ancienne sur le plan du renouvellement des méthodes, des problématiques, des perspectives. Peut-être l'un des renouvellements viendra-t-il ce 
que vous faites ici à Toulouse : étudier la réception de l'Antiquité. De ce point de vue, je pense qu'Anabases, et je ne dis pas ça du tout par flatterie à l'égard de mes collègues de Toulouse, apporte un vrai souffle nouveau, de la même manière que Topoi l'a fait à Lyon avec ses dossiers thématiques, ou ses recensions longues. Il s'agit à mon sens aujourd'hui des deux meilleures revues d'histoire ancienne, les plus novatrices, même si d'autres apportent évidemment leur lot d'informations utiles.

PASCAL PAYEN : Pour tenter d'expliquer ce phénomène, vous évoquez la fin des idéologies. Est-ce qu'il y aurait d'autres freins, par exemple institutionnels, dus à l'organisation même des universités?

Maurice Sartre: Oui, bien sûr. J'ai été suffisamment investi dans la gestion des universités au niveau national et local pour porter un jugement sur ce qui s'y passe. Le mode de fonctionnement actuel des universités françaises détourne les universitaires de ce qui est leur métier, c'est-à-dire de la recherche et de l'enseignement, pour les confiner à des tâches de gestion. Aujourd'hui, être directeur d'une unité de recherche c'est se condamner à ne plus faire de recherche. D'une manière générale, ce qui tue la recherche à l'université c'est le mode de fonctionnement des universités, où l'on doit concilier une réelle pratique démocratique (multiplication des conseils) et le dialogue permanent avec des tutelles omniprésentes. Pour rester dans le seul domaine de la recherche, comment ne pas être indigné par les propos présidentiels sur l'absence d'évaluation des chercheurs alors que nous passons notre temps à établir rapports et dossiers qui sont autant de moyens d'évaluation?

CORINNE BONNET : Je voudrais repartir D'Alexandre à Zénobie, paru en 2001 avec le soustitre Histoire du Levant antique, $v^{e}$ siècle avant - $\|^{e}$ siècle après. II a connu un succès considérable et tout à fait mérité. Vous brassez dans ce livre un espace-temps considérable et une documentation extrêmement diversifiée: inscriptions phéniciennes, palmyréniennes, ostraca araméens, sources nabatéennes, sud-arabiques, grecques et romaines... En exergue figure une phrase tirée d'un échange épistolaire entre Franz Cumont et Mikhaël Rostovtzeff que j'ai eu le plaisir de publier ${ }^{13}$; le 24 octobre 1937, Rostovtzeff écrit à Cumont: "Un livre de synthèse est toujours une torture, surtout quand il s'occupe d'une période peu connue et si peu compréhensible. » II s'exprime alors qu'il prépare son grand volume de synthèse publié en $1941^{14}$ : The Social and Economic History of the Hellenistic World. Cumont le rassure néanmoins en lui répondant: "Mais à quoi servent toutes nos recherches particulières, si elles ne doivent pas conduire à une synthèse? » Comment évaluez-vous les progrès de nos connaissances et les évolutions de nos grilles de lecture de l'histoire du Proche-Orient hellénistique et romain, depuis la synthèse de Rostovtzeff ? La rédaction d'une ample synthèse a-t-elle été, pour vous aussi, une sorte de torture? Et, plus généralement, Rostovtzeff fait-il partie de vos filiations intellectuelles?

Maurice Sartre: Vous m'obligez à des introspections auxquelles je ne suis guère habitué, parce que je suis plutôt du genre à avancer sans trop me poser de questions, je veux dire par là que je n'ai pas de stratégie « éditoriale ", publier une monographie plutôt qu'un manuel, par exemple. Mais c'est vrai que tout à l'heure, quand je regardais la pile de livres placée devant Pascal Payen, je n'ai pas réalisé tout de suite que c'étaient les miens, et que j'avais publié autant de livres. Évidemment, il ne s'agit pas exclusivement de livres d'érudition, car, même si l'érudition est indispensable, le discours purement érudit ne suffit pas pour séduire. Expression qui peut surprendre, mais dont je vais m'expliquer.

Pour ce qui est des synthèses, je les crois aussi indispensables qu'éphémères. D'autres chercheurs, plus jeunes, donneront un jour des synthèses proposant une nouvelle 
vision des sujets que j'ai traités. Et c'est très bien ainsi. Mais le devoir de synthèse est impératif, car il conditionne le renouvellement de la discipline; c'est ce que je nommais à l'instant "séduire»: donner envie à des jeunes de se lancer dans la recherche. Pourquoi beaucoup d'étudiants sont-ils venus me voir à Tours en disant «je veux faire une thèse avec vous »? Sans doute parce que j'avais écrit des choses qui leur donnaient envie de faire de la recherche, et c'est notamment pour eux que j'ai écrit. Il y a aujourd'hui une douzaine de jeunes chercheurs dans des universités et au CNRS qui ont fait leur thèse avec moi, qui ont eu envie de travailler sur des champs qu'ils avaient découverts en quelque sorte en lisant ce que j'avais écrit. Et où ils apportent des points de vue nouveaux et intéressants. L'auteur de synthèse n'est donc qu'un stimulant, mais son rôle est indispensable.

L'érudition signerait donc sa propre mort si elle ne débouchait pas, régulièrement, sur des synthèses qui provoquent l'envie de chercher chez les jeunes. La synthèse, c'est la tentative indispensable et impossible d'une explication globale. Mais l'écriture d'une synthèse provoque la souffrance, comme le notait Rostovtzeff, parce que le spécialiste d'un domaine, dans mon cas la Syrie du Sud, sait bien que, dès qu'il écrit sur tout le Proche-Orient, il ne possède pas le même niveau de connaissance érudite sur le Néguev, sur le Massif Calcaire ou la vallée de l'Euphrate. Donc il est en permanence sur la corde raide, en se disant « est-ce que là je ne suis pas en train de me tromper?", sachant que vous n'avez ni le temps de lire toute la littérature savante, et pas même l'ensemble des sources primaires, pour autant même que vous soyez capable de dépouiller avec efficacité et compétence le corpus des inscriptions sémitiques...

Donc la synthèse est doublement périlleuse. Parce que vous n'êtes pas le spécialiste de tout et que, là où vous l'êtes, vous n'êtes déjà pas toujours sûr de vos conclusions. Mais, en même temps, une synthèse est le lieu où vous pouvez donner un fil directeur, un souffle, une impulsion, une idée directrice. Que serait une synthèse qui se contenterait de faire l'inventaire de connaissances? Il y a, bien sûr, un aspect «inventaire », mais j'espère qu'il n'est pas le seul, qu'il y a derrière un certain nombre d'idées qui ressortent, que ce soient les vôtres en propre, ou qu'elles découlent des travaux savants dont vous vous faites le passeur. Vous ne pouvez pas dire à un étudiant de première année : «Mais, mon cher ami, allez donc lire Louis Robert. " Je le dis évidemment sans aucune moquerie à l'égard de Louis Robert qui reste, pour moi, le plus grand historien du siècle précédent. Un étudiant de première année ne peut évidemment pas lire Louis Robert: un mot sur deux est en grec, surtout dans les articles les plus anciens, et jamais traduit, cela allait de soi pour lui !

Pour moi, l'auteur de synthèse est donc aussi un médiateur. J'ai toujours considéré que ma mission, mon travail était de faciliter le passage des jeunes que l'on m'a confiés vers la science à laquelle je peux avoir accès. C'est pour cela que paradoxalement, quand je dis que j'ai écrit D'Alexandre à Zénobie pour moi, ce n'est pas une formule rhétorique : les informations sur la Syrie étaient très éparpillées et je ne trouvais aucun livre qui rassemblait tout ce que je savais ou croyais savoir sur le sujet. Certains prétendaient même que l'époque hellénistique restait très obscure (ce qui n'est pas entièrement faux), et qu'il n'y avait presque rien à en dire. Je me suis donc dit que ce livre dont j'avais besoin comme aide-mémoire, j'allais l'écrire moimême. J'aurais pu faire beaucoup plus, parce que 1200 pages c'est peut-être un peu court et qu'il y aurait bien d'autres choses à dire encore! Mais il faut bien tenir 
compte des possibilités des éditeurs! Je crois que, d'une certaine manière, on écrit toujours pour soi : le premier bénéficiaire d'un livre de synthèse, c'est son auteur. Même lorsqu'il s'agit d'un ouvrage d'érudition. Ainsi, les inscriptions grecques et latines de Syrie du Sud sont dispersées dans des dizaines de publications anciennes, souvent difficiles à trouver. D'avoir à en rédiger le corpus me permet de profiter en avant-première - et pour cause ! - et commodément de la totalité des inscriptions grecques de Syrie du Sud! Si écrire une synthèse revient à tenter de donner un sens à une masse d'informations disparates, la réalisation d'un corpus érudit n'est pas forcément de nature très différente : la rédaction en continu vous permet de déceler tout à coup des liens insoupçonnés entre des textes qui avaient été pris isolément. Prendre en compte une globalité, que ce soit un corpus régional d'inscriptions ou l'histoire synthétique d'une région, cela seul permet de donner du sens, d'abord aux yeux de l'auteur, parfois aussi pour d'autres à qui cela peut donner envie d'approfondir ce champ.

CORINNE BONNET: Quel écart sentez-vous par rapport à la vision qu'a Rostovtzeff de I'Orient grec et romain, que l'on qualifie souvent de « coloniale » ?

Maurice Sartre : C'est vrai que j'ai abandonné votre question en cours de route. Je ne crois pas que j'aie tellement d'écart avec Rostovtzeff... non pas en ce qui concerne le talent, qui est immense chez lui, mais en ce que je m'inscris dans le fil de sa volonté de comprendre en bloc. Il a fait ces deux livres admirables ${ }^{15}$, même si l'un des deux me paraît aujourd'hui plus périmé que l'autre ${ }^{16}$, et je pense que, si un certain nombre d'études sont en cours sur le Proche-Orient aujourd'hui, c'est bien grâce à Rostovtzeff, et sa lecture reste passionnante et indispensable. En le lisant, j'ai eu envie de creuser davantage. Je me situe un peu dans la même ligne; c'est pour ça qu'aujourd'hui je considère qu'il manque un peu, dans l'école historique française, d'auteurs de synthèses - pas forcément des synthèses générales, mais des synthèses thématiques, des livres destinés à un grand public. J'aimerais que les historiens plus jeunes que moi prennent leur courage à deux mains et se lancent dans des synthèses.

Cela dit, je me situe probablement dans une perspective idéologique très différente de Rostovtzeff. Non seulement je n'ai pas vécu la Révolution russe, mais j'ai même écrit mes diverses synthèses après la chute du régime qu'elle avait mis en place. Je n'ai donc pas été obsédé par la question des bourgeoisies naissantes et de leur capacité à entreprendre. J'ai davantage été sensible aux questions de culture, de métissage et d'intégration, de transferts culturels et de modification des mœurs. Mais ne soyons pas dupes: ce faisant, je suis aussi sensible aux préoccupations de mon époque que Rostovtzeff l'était à celles de la sienne! On l'accuse d'une vision trop « coloniale », alors qu'il écrit à l'apogée des Empires coloniaux; n'est-il pas naturel qu'on écrive une histoire moins coloniale vingt ou trente ans après la fin des mêmes Empires? Mais la préoccupation qui est la mienne - et celle de bien d'autres - de comprendre l'interpénétration des cultures, les voies de l'intégration des individus dans une culture qui n'est pas la leur, ou l'importance des transferts culturels découle au moins en partie de l'époque que vous vivons, celle du « choc des civilisations » (il va de soi que je n'adhère ni de près ni de loin à cette ânerie) et de la mondialisation, avec ce que cela implique de frictions, de difficultés, de rejets de l'autre.

CORINNE BONNET : Dans la salle où nous nous trouvons, a eu lieu un débat autour du livre contesté de Shlomo Sand sur la formation de l'identité d'Israël17. Or, dans votre livre, comme dans bien d'autres, l'histoire de la Palestine hellénistique et romaine fait l'objet d'un chapitre approfondi où vous mettez bien en relief la diffıcile rencontre entre judaïsme et 
hellénisme. Dans quelle mesure à vos yeux I'histoire de la Palestine est-elle singulière? Dans quelle mesure peut-elle être écrite sur le registre de la continuité ou de la discontinuité, en tenant compte du fait que, comme toutes les autres «nations » de la région, elle a été hellénisée, intégrée dans l'empire d'Alexandre, puis dans l'empire romain, etc. ?

Maurice Sartre : Je connais bien le livre de Shlomo Sand, parce que j'ai participé à plusieurs débats, et je ne suis pas du tout d'accord avec les critiques virulentes qui ont été faites de ce livre. Je l'ai dit, je l'ai écrit, notamment dans le dernier numéro de la revue Le Débat ${ }^{18}$. Le livre de Shlomo Sand est très irritant parce que bourré d'erreurs factuelles, de petites approximations, ce qui a justifié de la part de ses adversaires, qui s'attaquaient à lui pour des raisons idéologiques, qu'il soit traîné plus bas que terre. Il n'en reste pas moins qu'en dépit de ces erreurs, évidemment regrettables, le livre de Shlomo Sand est, d'une manière générale, fondamentalement juste. Il met le doigt sur deux choses essentielles à mes yeux d'historien. D'abord, sur le fait qu'il y a eu des mouvements de conversion au judaïsme importants - il dit « massifs », je préfère le terme « importants »-, dans l'Antiquité. Donc une partie des juifs de ce temps sont des « convertis ». Or, c'est aujourd'hui quelque chose qui est totalement nié par les milieux les plus conservateurs ou les plus violemment sionistes, puisque cela remet en cause l'idéologie même sur laquelle se fonde l'État d'Israël, c'est-à-dire le don de la terre d'Israël par Dieu à un peuple prédéfini. D'autre part, ce que dit très bien Shlomo Sand, c'est que la plupart des juifs de Palestine se sont évidemment convertis au christianisme puis, comme presque tout le monde, à l'islam; au point que - Ben Gourion le disait lui-même sous forme de pseudo boutade - les vrais descendants des juifs de l'Antiquité sont peut-être en réalité les Palestiniens d'aujourd'hui. Il ne va pas jusque-là, mais évoque néanmoins cette possibilité, à titre de provocation. Il a, en outre, parfaitement raison de montrer qu'il existe toute une historiographie juive du XIX ${ }^{e}$ siècle, qui s'inscrit très exactement dans la perspective des nationalismes de l'époque: c'est alors qu'on invente Vercingétorix en France, que les Allemands se fondent autour d'Arminius et des victoires contre les Romains. Les juifs aussi ont à ce moment-là, tout d'un coup, découvert qu'ils étaient un peuple et ils se sont racontés comme tel, ce qui était facile puisqu'ils pouvaient s'appuyer sur la Bible à laquelle même les chrétiens croyaient. C'est donc un livre tout à fait passionnant, malgré l'irritation que l'on peut ressentir à sa lecture, en raison des erreurs et des excès.

Pour le reste et pour revenir à l'Antiquité, il ne faut pas perdre de vue deux choses. En premier lieu, les juifs sont une entité spécifique, qui tranche sur tous les autres peuples du Proche-Orient sur le plan religieux, par leur monothéisme. Mais plus encore, c'est par leur exclusivisme qu'ils se singularisent. Cela ne gêne personne que les juifs ne croient qu'à un seul dieu, mais ce qui les distingue de tous les autres et les rend insupportables aux yeux de beaucoup, c'est qu'ils sont les seuls au ProcheOrient et autour de la Méditerranée à ne pas vouloir admettre que les dieux des autres sont aussi des dieux. Les Phéniciens, par exemple, savent très bien que les gens de Palmyre ou de Damas ont leurs propres dieux, et réciproquement, et s'ils ne les vénèrent pas, ils n'en considèrent pas moins que ce sont des dieux comme les leurs. D'ailleurs, les échanges et emprunts ne sont pas rares. Les juifs au contraire nient le caractère divin des dieux des autres.

Une fois clairement affirmée cette spécificité, on ne doit pas négliger un second fait : sur le plan de la société et des comportements, les juifs ne sont pas un isolat. Ils 
vivent dans une petite région, de la Judée à la Syrie du Sud, et y cohabitent avec d'autres, sans parler des villes de Syrie où se trouvent de nombreuses communautés exilées. Pour ma part, je ne vois pas comment on peut écrire une histoire du ProcheOrient d'où les juifs seraient absents, de la même manière que je refuserais d'écrire une histoire des juifs où il n'y aurait pas les autres. Mais cela ne plait pas à tout le monde : mon livre sur le Proche-Orient attendra sans doute longtemps avant d'être traduit en arabe, car certains trouvent que j'y parle trop des juifs !

Depuis la parution de ce livre, dans un volume de mélanges en l'honneur de Glen Bowersock en 2006, j'ai proposé un texte intitulé « De Pétra à Jérusalem... et retour ${ }^{19}$ ». J'ai voulu montrer dans cet article que le royaume nabatéen et le royaume hasmonéen évoluaient de façon étrangement parallèle. Par exemple, ils adoptent l'un et l'autre une monnaie d'État à dix ans d'intervalle à peine; leurs souverains prennent le titre royal presque en même temps et se mettent à copier les institutions des royaumes hellénistiques à peu près en même temps. Donc je crois qu'il ne faut pas se tromper: les juifs sont dans la société de leur temps, quel que soit leur exclusivisme. J'irai même plus loin. Je suis persuadé que l'espèce de radicalisation légaliste (on parlerait aujourd'hui de délire fondamentaliste) à laquelle on assiste dans le courant du $\mathrm{I}^{\mathrm{er}}$ siècle de notre ère chez les juifs de Judée - c'est l'époque où les rabbins essaient de faire appliquer par l'ensemble de la société les règles de pureté, les règles de la Torah, y compris celles qui étaient réservées à l'origine aux prêtres -, ne s'explique que parce que les chefs spirituels du judaïsme, les rabbis, sentent l'ensemble de la société juive sur le point de leur échapper. Cette société juive, en contact avec la culture grecque, c'est-à-dire, j'emprunte le mot à Paul Veyne ${ }^{20}$, avec la culture "moderne ", depuis trois ou quatre siècles, et dont la moitié au moins vit au contact quotidien des Grecs dans la diaspora, qui existe depuis bien avant la destruction du temple en 70 - un autre point où Shlomo Sand a raison -, est perçue par les rabbins comme prête à basculer dans le " modernisme », dans les délices de la société de leur temps. On érige alors la Torah comme une barrière autour de la communauté juive, on essaye de monter le mur des interdits le plus haut possible. On en rajoute, et c'est là que prennent de l'ampleur les discussions juridiques les plus pointues, les plus insensées en apparence, cette érudition légaliste qui traduit la diversité des opinions dans un âge d'angoisse face à l'avenir. Les Talmuds sont rédigés ultérieurement mais reprennent souvent des discussions remontant au $\mathrm{I}^{\mathrm{er}}$ siècle. On se pose alors des questions qui nous semblent extravagantes, voire futiles : est-ce qu'une femme qui avait un grain de sel ${ }^{21}$ dans la bouche le jour du shabbat et qui laisse le grain de sel tomber a le droit de le ramasser, doit-elle en prendre un autre, ou s'en passer ? C'est dire qu'on considère que tout aspect, même mineur, de la vie de chacun est susceptible de porter atteinte au respect que chacun doit à la Torah.

Il en va de même de certaines anecdotes que raconte Flavius Josèphe. Ainsi, des juifs pieux sont prêts à se faire égorger pour protester contre les soldats romains entrés à Jérusalem de nuit en cachant les enseignes de leur unité sous des chiffons, afin de ne les sortir qu'une fois arrivés au camp ; même cachés, les enseignes offusquent leurs yeux qui ne les voient pas! Une autre fois, des juifs accusent Hérode d'avoir fait représenter des êtres vivants sur un mur du Temple et de les avoir masqués par des parements de bois et de pierre. On démonte donc les pierres et l'on s'aperçoit que derrière il n'y a rien de ce qui était dénoncé. Rien n'y fait, ils étaient furieux au départ, ils restent furieux après. Cette rage fondamentaliste - difficile de ne pas 
songer aux plus extrêmistes des taliban - s'explique, je crois, par la crainte des rabbins de voir la communauté basculer dans le camp du modernisme; c'est un aspect d'une crise identitaire grave, et je me demande parfois si ce n'est pas un peu ce que vivent certains fidèles dans l'islam d'aujourd'hui, mais c'est un autre sujet.

Je crois donc qu'il ne faut jamais isoler les juifs quand on écrit l'histoire du ProcheOrient, tout en n'oubliant pas leur spécificité : elle est évidente. Et, une fois passées les crises violentes qui ponctuent l'histoire de la Judée, des Maccabées à Bar Kokhba, ils s'intègrent plus volontiers. Ainsi, on en trouve jusque dans des thermes où trône le portrait d'Aphrodite : c'est la superbe anecdote de la discussion entre Raban Gamaliel et l'imaginaire Proclos Ben Philosophos que j'ai racontée dans une séquence des Histoires grecques ${ }^{22}$. Le Talmud raconte que Raban Gamaliel, on ne sait pas lequel (il y en a au moins deux) mais ça n'a pas d'importance, la plus haute autorité du judaïsme - c'est un des patriarches du judaïsme -, se trouvait un jour aux thermes, à Ptolémaïs. S'approche de lui un Grec, qui a un nom d'emprunt, Proclos, comme plusieurs philosophes, Ben Philosophos, nom inventé pour l'occasion, qui lui dit en résumé : "Mais enfin, toi, un juif, tu viens aux thermes alors qu'il y a là une statue d'Aphrodite !» Il faut rappeler que quelques siècles auparavant, lorsque Jason, grand prêtre de Jérusalem, a construit un gymnase dans la ville, où, pourtant, il n'avait pas mis les images d'Hermès et d'Héraclès, il s'était fait accuser d'impiété.

Raban Gamaliel répond alors à Proclos qu'ils parleront de cela dehors, parce que l'on ne parle pas de choses sérieuses aux thermes. Ils sortent donc, et Gamaliel lui dit: «Oui, je viens aux thermes, comme tout le monde; quant à Aphrodite, ce n'est pas moi qui vais chez elle, c'est elle qui est venue chez moi.» "Que veux-tu dire par là ? », demande Proclos. Gamaliel lui répond : "T'arrive-t-il d'aller uriner devant la statue de tes dieux? " "Bien sûr que non. Pourquoi cette question?", rétorque le philosophe. «Tu as vu où est la statue d'Aphrodite? Elle est à l'endroit où nous allons tous uriner! Ce n'est donc pas une statue de culte: c'est un décor ", conclut Raban Gamaliel.

Il fait ainsi prendre conscience à Proclos du fait qu'il y a statue et statue. Il y a celle qui représente la divinité dans son temple, qui est une statue de culte, qui est donc sacrée. Et puis il y a celle qui relève d'une catégorie neutre, le décor, qui est là pour faire joli.

L'anecdote est intéressante surtout parce qu'elle montre que, lorsqu'il faut s'adapter au monde moderne, on fait la part entre l'accessoire et l'essentiel : l'essentiel, c'est de protéger une vie juive là où les Juifs sont devenus minoritaires, comme en Palestine dès le début $\mathrm{du} \mathrm{II}^{\mathrm{e}}$ siècle, dans beaucoup de régions, peut-être à l'exception de la Galilée. $A u{ }^{\mathrm{er}}$ siècle de notre ère, aucun rabbin ne serait allé aux thermes à Césarée ! On ne fréquentait même pas le stade, parce qu'il y avait des statues, qui étaient forcément considérées comme des idoles. Lorsqu'un siècle plus tard, les juifs sont devenus minoritaires, l'intelligence extraordinaire des rabbins est d'inventer le discours théorique qui permet de continuer à vivre et de dire qu'il faut respecter tous les principes sacrés des juifs. Par exemple, il est interdit d'honorer les statues de culte, de même qu'il est interdit de manger la viande sacrifiée aux idoles. Mais il y a des statues qui relèvent du décor: c'est pour cela que l'on trouve à Sépphoris des maisons juives avec des représentations de Dionysos. 
Il y a donc une formidable adaptation du judaïsme, qui est la condition de sa survie. Cette anecdote montre aussi que, malgré tout, les juifs vivent très bien avec les autres ; dès lors, écrire une histoire des juifs seuls n'a pas de sens, au contraire d'une étude qui embrasse l'ensemble des populations du Proche-Orient. C'est ma conviction profonde.

PHILIPPE FORo : L'année dernière, lors de la journée sur Jérusalem, vous aviez parlé de la révolte des Maccabées et vous aviez montré qu'elle reflétait une coupure entre des juifs qui participaient à la culture grecque et d'autres qui s'en sentaient exclus.

Maurice Sartre : En effet, c'est là qu'est le vrai problème. Je ne vais pas pousser trop loin le parallèle, mais je crois que la crise que vit l'islam aujourd'hui relève un peu de la même logique. C'est-à-dire qu'il y a ceux qui ont envie de se fondre dans une société moderne et ceux qui s'en sentent rejetés ou qui pensent que se fondre dans le monde moderne ne peut se faire qu'au prix de la perte de leur identité profonde, qu'ils estiment liée au maintien intégral de leurs traditions. La grande difficulté des juifs de l'Antiquité, c'est qu'ils ont mis trois ou quatre siècles à comprendre qu'on pouvait rester juif et devenir grec en même temps. À l'époque des Maccabées, la plupart des juifs pense que l'on doit faire un choix : être soit grec, soit juif, les deux paraissant totalement antagonistes pour des raisons notamment religieuses. Rares sont alors ceux qui tentent l'aventure de rester juif en devenant grec. La réforme de Jason à Jérusalem (175 av. J.-C.) ne met absolument pas en cause l'appartenance au judaïsme, du moins consciemment. Celle de Ménélas est sûrement plus radicale et donc plus discutable, mais Jason ne veut, en quelque sorte, que moderniser, inciter les juifs à se conduire comme les autres lorsque cela ne parait pas remettre en cause leur fidélité à Yahvé. Il dit aux juifs : conservons notre dieu unique et allons au gymnase - et, de fait, la Bible n'a jamais interdit le gymnase. Bien sûr, la nudité sportive heurte la pudeur traditionnelle, mais là où tout bascule, c'est lorsque les juifs qui vont au gymnase et pratiquent le sport nus masquent la circoncision et l'abandonnent pour leurs enfants. Alors, mais alors seulement, ceux-ci violent des règles fondamentales du judaïsme.

Donc, effectivement, la difficulté est d'adapter traditions et modernité, d'être capables d'analyser quelles traditions sont fondamentales sur le plan religieux, et celles qui ne sont que des héritages d'autres modes de vie correspondant à une époque donnée. À l'inverse, il convient de trier dans les emprunts au monde moderne ce qui est compatible avec la vie juive et ce qui ne l'est pas. Les juifs pouvaient très bien aller courir nus au gymnase en étant circoncis. Les Grecs en étaient choqués, parce que c'était une atteinte à l'intégrité corporelle, mais ils ne leur interdisaient pas pour autant. L'idée est donc de comprendre que les cultures ne sont pas exclusives les unes des autres. Elles peuvent se combiner, se mélanger ; ceux qui sont spécialistes de l'Afrique ou de la Gaule, par exemple, le savent bien. Ce n'est pas parce que l'on se « romanise » que l'on abandonne sa culture originelle. Les cultures sont toujours mixtes. Les juifs ont mis quatre siècles à comprendre qu'ils pouvaient être juifs et grecs.

PHILIPPE Foro : De ce point de vue, le christianisme vous semble-t-il différent? II n'a pas connu ce type de débats?

Maurice Sartre : Le christianisme n'a pas à avoir ce débat, parce qu'il n'est pas fondé au départ sur une ethnicité. Il ne faut pas oublier que ceux que nous appelons les «juifs» sont, pour les Grecs, hoi ioudaioi, c'est-à-dire les Judéens ${ }^{23}$. Ils raisonnent 
comme des Romains : on est originaire d'un endroit, donc il existe les dieux de cet endroit, les mœurs de cet endroit, la cuisine de cet endroit... Pour les chrétiens, la question de l'ethnicité ne se pose pas à l'origine, du moins dès qu'est admise la conversion des païens par Paul de Tarse. La question de la culture apparaît néanmoins plus tard, en réponse aux païens sur la défensive, lorsque Julien l'Apostat leur dit que, puisqu'ils ne croient pas aux dieux mentionnés dans les textes antiques, les chrétiens ne peuvent plus enseigner les auteurs classiques. C'est le fameux décret de juin $362^{24}$, qui, fût-il encore appliqué, signerait la mort des professeurs d'histoire grecque : lequel d'entre nous vénère encore Zeus ou Athéna? Les chrétiens doivent alors se défendre, en rappelant qu'il s'agit de leur propre culture autant que celle des païens. Il faut lire et faire lire l'admirable défense de Basile de Césarée, Lettre aux jeunes gens sur le bon usage des lettres helléniques, qui explique en quoi la culture grecque est le bien commun à tous, y compris des chrétiens. Mais il est vrai que la vision de l'homme de culture qu'est Basile n'est pas nécessairement partagée par les moines obscurantistes qui abattent les temples et brûlent les manuscrits « idolâtres ».

PUBLIC : Les Romains faisaient-ils une différence entre les peuples sémitiques ? Vous avez parlé des juifs, mais vous n'avez jamais parlé des autres peuples sémitiques.

Maurice Sartre : Bien sûr qu'ils font des différences. Il y a une chose que les Romains n'utilisent jamais, c'est le terme d'Oriental, sauf pour désigner un ensemble géographique, c'est-à-dire ce qui est à l'Est. Le terme me hérisse toujours dans les travaux de mes collègues ou de mes étudiants. Un Oriental, ça n'existe pas et je ne sais pas ce qu'est une culture orientale. Les Grecs, comme les Romains, font très bien la différence entre les Phéniciens, les Syriens, les Chaldéens, les Cappadociens, les Arméniens ; cela ne veut pas dire qu'ils ne les confondent pas parfois, mais ils savent généralement les distinguer, quitte à les qualifier de manière profondément « raciste », c'est-à-dire globalisante à l'échelle d'un peuple : les Arabes, nomades et brigands (d'ailleurs c'est synonyme), les Égyptiens, les Phrygiens, les Ciliciens... Ils font parfois des confusions entre eux, mais ils n'ont jamais globalisé à l'échelle du Proche-Orient : ils ne connaissent pas du tout le vocable de "Sémites ", évidemment, et n'ont pas laissé de textes qui montreraient qu'ils ont conscience de la parenté linguistique entre les divers peuples sémites. Il faut dire que l'emploi quasi général de l'araméen en Syrie et Mésopotamie masque en partie les nuances régionales.

ÉLODIE GUILLON : Je voudrais évoquer Maurice Sartre l'archéologue, qui n'est pas très éloigné de Maurice Sartre I'historien, puisque vous êtes un auteur de synthèses, un médiateur - vous l'avez dit -, et que vous résumez tout cela dans une formule, dans un compte rendu d'un ouvrage de Pierre Leriche en 1987: "La fouille n'est qu'un moyen d'écrire l'histoire ${ }^{25}$. » Depuis une trentaine d'années s'opère un renouvellement des questionnements en archéologie, et la tendance la plus récente consiste à replacer les hommes dans leur environnement, soit physique (les interactions entre hommes et territoires), soit symbolique et culturel. Vous avez commencé l'archéologie très tôt, en Afghanistan, en 1968, puis, en 1969, en Syrie. Vous êtes donc un témoin privilégié de l'évolution de la discipline, puisque vous ne l'avez jamais abandonnée, ainsi qu'en témoignent vos dernières publications. Ma première question est liée à la lecture d'un ouvrage que vous avez préfacé : les actes d'un colloque international qui s'est tenu à Tours sur l'Asie Mineure dans l'Antiquité, parus en 200926. Vous y faites écho aux questionnements très actuels sur la perception du territoire, l'espace civique et les identités. Les contributions sont celles de jeunes chercheurs et l'on pourrait s'attendre à ce que l'archéologie y ait la part belle. Or, il n'en est rien: seule une intervention utilise réellement les nouveaux outils et les nouvelles questions de l'archéologie. Les autres font une utilisation presque désuète de la source archéologique, qu'ils utilisent comme 
illustration d'un discours historique déjà bien construit. À votre avis, existe-t-il, dans les études actuelles une sorte de contraste entre les avancées de l'archéologie et l'utilisation que l'on en fait dans l'écriture de l'histoire? Ce contraste est-il typique des travaux français?

Maurice Sartre: Tout d'abord, je ne me présenterais sûrement pas comme un archéologue. Il est vrai que j'ai eu la chance d'aller fouiller en Afghanistan, en 1968 j'étais très jeune et cela m'a donné l'occasion unique de voir un peu l'Afghanistan -, je ne suis donc pas complètement étranger à l'archéologie ; par la suite j'ai fait de la prospection de terrains, des surveys ce qui est tout à fait différent, avec une orientation nette vers l'épigraphie et la recherche des inscriptions.

Cela dit, je crois que vous avez raison : l'archéologie sert trop souvent, aujourd'hui encore, d'alibi, d'illustration d'un discours historique déjà construit. Les responsabilités sont partagées entre les historiens et les archéologues. Par ses méthodes, l'archéologie est une discipline exigeante, difficile, au niveau requis de compétence technique de plus en plus élevé ; mais, dans le même temps, ce n'est pas une fin en soi : si l'archéologie ne sert pas aussi à écrire l'histoire, je ne sais pas à quoi elle sert. Et, malheureusement, trop de publications archéologiques sont pratiquement inutilisables par d'autres que ceux qui ont fouillé. Or, quand vous écrivez une synthèse, vous devez vous poser toutes les questions possibles et imaginables, y compris celles sur lesquelles on n'a jamais rien écrit. Par exemple, que sait-on sur la céramique comme témoin des échanges en Méditerranée au $\mathrm{III}^{\mathrm{e}}$ siècle avant J.-C. ? Si vous vous posez la question, vous entreprenez de lire tout ce que vous trouvez là-dessus, et parfois vous ne comprenez rien soit parce que les archéologues ont écrit pour eux seuls, soit parce que cela ne débouche sur aucune conclusion claire. Aujourd'hui, on souffre d'un double problème : trop d'archéologues ne savent plus pourquoi ils fouillent et donc, lorsqu'ils exposent les résultats de leurs recherches, ils ne les rattachent à aucune problématique claire ; et, en conséquence, les historiens n'utilisent l'archéologie qu'au moment où ils ont besoin d'illustrations pour leurs propres discours.

Comment en sortir? Tout d'abord, avoir des archéologues qui ont fait des études d'histoire, je trouve que c'est une bonne chose. Il y a encore une vingtaine ou une dizaine d'années, les archéologues au CNRS ne voulaient surtout pas être dans la même section que les historiens de l'Antiquité ! Et le phénomène persiste dans certaines universités aujourd'hui. Tout de même, les fouilles les plus classiques (villages, villes, monuments, céramique, monnaies...), restent le fondement même de l'étude historique de l'Antiquité. Il y a une solidarité de l'archéologue et de l'historien pour l'époque antique. Je crois, en outre, que tout ce qui est étude des paysages, prospections étendues sur de multiples sites sur l'ensemble d'une région, etc. est très prometteur ; c'est même le seul moyen dont on dispose pour retrouver la trace des plus modestes, de ce qui est le plus ténu. Voyez par exemple le livre, paru voici quelques années, sur les nomades d'Asie centrale ${ }^{27}$ : pas facile, l'archéologie du nomadisme, avec quelques trous de poteaux; les nomades de l'Antiquité ne laissent que peu de traces. Cette archéologie permet de reconstruire un modèle d'environnement ; et c'est, à mon avis, très intéressant.

ÉLODIE GUILLON : Ma première question était un peu biaisée puisque je n'y ai pas mentionné le rôle de pôles très dynamiques, comme l'Institut français du Proche-Orient ${ }^{28}$, qui mène sur place de grands programmes cohérents. Vous êtes lié à l'ifpo et à son réseau doctoral. J'aimerais avoir votre avis sur la place de ce pôle dans la chaîne de production des savoirs 
archéologiques: élaborent-ils de nouvelles problématiques? utilisent-ils de nouvelles techniques? ou bien ne sont-ce que des vitrines?

Maurice Sartre : Selon moi, ce ne sont pas des pôles moteurs, ni tout à fait des vitrines. Les vrais moteurs de la recherche aujourd'hui, ce sont les équipes de recherche. C'est de là et de là seulement que peuvent venir les vraies initiatives sur des problématiques variées. L'IfPo est une structure lourde maintenant, puisqu'il regroupe l'Institut français d'archéologie au Proche-Orient, l'Institut français d'études arabes de Damas et le Centre d'étude et de recherche sur le Moyen-Orient contemporain, installé à la fois à Damas, à Beyrouth et à Amman. Mais cet ensemble est plutôt une structure d'accueil de chercheurs qu'une structure d'impulsion en matière de recherche. Au total, c'est une vitrine à l'égard des autorités locales pour marquer notre présence et notre intérêt envers les pays dans lesquels nous sommes installés et c'est une structure de collaboration avec les chercheurs locaux, comme l'illustre le comité de suivi rassemblant chercheurs de l'IFPo et autorités des pays d'accueil pour élaborer ensemble des projets.

Mais l'IFPo a peu de programmes de recherche propres, pour l'Antiquité, généralement en collaboration et cela s'explique notamment par la rotation rapide des chercheurs. Il soutient des programmes, comme les inscriptions grecques et latines de Syrie (IGLS), comme le programme sur les temples et sanctuaires du ProcheOrient. Il sert aussi d'interface entre les missions françaises au Proche-Orient et les autorités locales. Il doit y avoir actuellement dix-neuf missions archéologiques françaises en Syrie. L'IFPo facilite l'obtention des permis, la régularité des papiers, etc. L'IfPo n'a pas de réel rôle d'animation scientifique ; on peut le regretter, mais il faut aussi remarquer que la culture, depuis deux-trois ans notamment, n'est plus vraiment à la mode. Il a d'autre part un rôle de diffusion, par les publications, malgré les difficultés financières de ce secteur. On ne sait pas bien à quoi servirait la recherche si on ne pouvait plus la publier; on est actuellement obligé de trouver des sponsors, mais ce qui est possible pour des ouvrages de numismatique ou les bijoux antiques, l'est un peu moins pour les inscriptions grecques...

L'IFPo joue également un rôle important dans le réseau doctoral. Mais le système repose plus sur des personnes que sur l'institution elle-même. Le réseau doctoral lie un certain nombre d'universités françaises et l'IFPO; à trois reprises déjà, on a organisé des semaines doctorales à Damas, Amman et Beyrouth, où l'on fait venir l'ensemble des jeunes chercheurs travaillant sur le Proche-Orient autour d'un thème donné, comme l'eau. L'initiative est importante, parce qu'elle permet aux jeunes chercheurs dispersés en France de se rencontrer sur le terrain et de prendre contact avec les réalités locales. On organise également des rencontres entre les chercheurs en poste à l'IFPo et les doctorants syriens et d'ailleurs. L'IFPO a, à mon avis, un rôle important à jouer dans le développement de ces échanges. Il n'en reste pas moins que l'IFPo aujourd'hui manque de crédits pour jouer pleinement son rôle d'interface. Ce n'est donc pas des programmes-mêmes de l'ifPo que viendra l'innovation.

PUBLIC : Depuis quand cette institution ne s'appelle-t-elle plus IFAPO?

Maurice Sartre : Depuis 2003. La fusion a été effective le $1^{\mathrm{er}}$ janvier 2003. Mais l'IFAPO lui-même n'était pas très vieux (1976). Avant il s'appelait l'IFAB, Institut français d'archéologie de Beyrouth, depuis sa fondation en janvier 1945. 
ÉLODIE GUILLON : Une dernière question, sur le domaine particulier de l'archéologie rurale, dont vous utilisez les sources dans votre ouvrage D'Alexandre à Zénobie. Au Proche-Orient c'est un domaine extrêmement novateur, qui apporte beaucoup de connaissances, d'autant plus que les fouilles urbaines posent problème, puisque les sites sont occupés depuis des millénaires et, à moins de destructions, comme à Beyrouth pendant la guerre, on ne peut fouiller. C'est aussi dans ce domaine que sont testées de nouvelles techniques, par exemple dans un grand programme sur la Syrie du Sud, coordonné par Pascale ClaussBalty, où l'on utilise l'outil informatique et les systèmes d'information géographique $(\mathrm{SIG})^{29}$. Quel est votre avis sur l'avenir de l'archéologie rurale? Va-t-elle perdurer comme domaine novateur ou rencontrera-t-elle ses limites, d'une part, parce qu'il y a des régions très bien connues archéologiquement et d'autres pas du tout, d'autre part, parce que, lorsqu'on étudie un territoire, arrive un moment où il est nécessaire de disposer de vestiges urbains? Ou bien, pour finir, le domaine va-t-il se modifier en profondeur - aujourd'hui on fouille peu et on prospecte beaucoup?

Maurice Sartre: Il est compliqué de répondre à cette question. Je pense que le potentiel archéologique de la Syrie est énorme, mais il y a plusieurs problèmes. Tout d'abord, il existe des études fort bien faites - vous avez cité le nom de Pascale ClaussBalty, et c'est une honte qu'elle ne puisse continuer ce travail faute d'avoir été recrutée sur un poste en France après son séjour à Damas -, mais on relève la disparition accélérée de ce potentiel en raison du développement des villages. Leur nombre et leur étendue se sont, en effet, accrus dans le sud de la Syrie depuis une quarantaine d'années. Lorsque je venais dans le Hauran il y a quarante ans, les villages étaient de petites grappes de maisons noires en basalte, serrées les unes contre les autres, sur un petit tell. Aujourd'hui, ce sont des villes pratiquement continues, les villages se touchent les uns les autres, le béton est partout. Des études, comme les prospections aériennes, déjà impossibles pour des raisons de sécurité, n'auront de toute façon plus d'intérêt. Heureusement que l'on a la couverture photographique aérienne de la Syrie à l'époque du mandat ! Ce qui serait urgent, ce serait de refaire ou d'utiliser les photos aériennes faites à différentes périodes.

Ce qui est plus grave, c'est que l'on a la chance d'être dans un pays où les vestiges audessus du sol sont souvent conservés, mais que ces élévations disparaissent en raison de la frénésie de construction. On démolit les maisons antiques (et l'on a dans certains villages des maisons antiques où il ne manque que le toit: les façades, les plafonds du premier étage, les escaliers extérieurs, etc. sont tous conservés), pour faire les fondations de maisons en béton que l'on va construire au-dessus.

Il y a donc là une urgence absolue à faire des relevés, à faire des photos, à faire le travail qu'a mené Pascale Clauss-Balty sur les maisons. Le mur de la maison de Muhajjeh qu'elle a repéré, où se trouvaient trois épigrammes funéraires que j'ai publiées avec Cécile Pouderon ${ }^{30}$, a disparu, alors que la maison était intacte il y a encore trois ans.

Cela dit, il ne faut jamais totalement désespérer. Il y a maintenant quelques oNG syriennes placées sous le patronage d'Asma El-Assad, l'épouse du président, femme extrêmement cultivée, qui prennent en charge des choses aussi diverses que les handicapés ou l'archéologie. On va donc essayer de sauver ce qui peut encore l'être. Le potentiel reste gigantesque, mais jusqu'à une date récente la démographie galopante en Syrie du Sud (souvent treize enfants) entraînait un énorme besoin de construction. On est maintenant tombé à environ cinq enfants en moyenne, mais les émigrés partis travailler dans le Golfe rapportent de l'argent avec lequel ils font construire une belle maison en béton. Et les maisons antiques se trouvent noyées, 
littéralement, dans cette masse de cubes de béton, quand elles ne sont pas tout simplement détruites.

ÉLODIE GUILLON : Aurions-nous besoin d'images de près ou plutôt d'images aériennes pour ces relevés?

Maurice Sartre : Il faudrait tout: prendre des mesures, faire des relevés précis au sol. Nous avons les moyens techniques, mais il faut avoir l'autorisation de les utiliser ! La Syrie reste un pays officiellement en guerre avec son voisin, et cela entraine de nombreuses interdictions matérielles, ou du moins de sérieuses limitations. Cela peut sembler absurde car Internet fonctionne et avec Google Earth on voit tout, y compris sans doute des sites militaires... ! Il faudrait surtout avoir beaucoup plus de monde à l'ouvrage : j'ai visité entre trois cents et quatre cents villages en Syrie du Sud, et il n'y en a pas un où il n'y ait rien à enregistrer.

NATHAËL RECOURSÉ : J'aimerais repartir de plusieurs choses que vous avez dites, qui me semblent intéressantes pour réfléchir sur les relations entre Proche-Orient ancien et Proche-Orient contemporain. Vous avez travaillé sur la notion d'identité, et je m'interrogeais sur le processus d'élaboration de cette identité. La prise en compte de l'Antiquité est-elle un moyen, pour les États contemporains, de se doter d'une identité bien précise? Comment, dans des pays comme la Turquie, la Syrie, le Liban, ou encore Israël, l'intégration de I'héritage antique se fait-elle? Et cette intégration, si elle existe, participe-t-elle de l'élaboration d'une certaine identité nationale, qui pourrait être mise en avant lorsqu'il s'agit d'aborder les grands enjeux géopolitiques du monde contemporain, comme le panarabisme du Ba'ath syrien par exemple?

Maurice Sartre: Je commencerai par deux citations. Benedetto Croce: «Il n'y a d'histoire que contemporaine ${ }^{31} »$; Yves Lacoste : « La géographie sert d'abord à faire la guerre ${ }^{32}$. » On peut en dire autant de l'histoire. Il ne faut pas se faire d'illusion: nous ne sommes pas des isolats, vivant dans un temps "intemporel», dans une époque sans âge. Ce monde, qui est le nôtre, a une influence sur nous. Ma manière de faire de l'histoire ancienne est fortement marquée par le monde dans lequel je vis. Je n'ai aucune illusion sur le sujet, ni aucune honte. Je ne veux pas dire par là que tout ce que j'écris est biaisé, mais que forcément les questions que je me pose sont des questions qui me sont dictées par le monde dans lequel je vis. Vous avez dit que j'ai beaucoup parlé ces dernières années des problèmes d'identité. De fait, ces problèmes sont au cœur des sociétés européennes d'aujourd'hui. Ce sont les problèmes d'intégration des émigrés, de l'intégration ou de la cohabitation dans une même unité, l'Europe, de peuples divers. Il faut donc être parfaitement lucide là-dessus : nous sommes forcément orientés dans nos recherches par les questions du monde contemporain.

Cela dit, les réponses que nous apportons aux questions historiques doivent être fondées sur la documentation qui est la nôtre. Lorsqu'un collègue israélien fait un livre sur la Syrie du Sud et les États hérodiens, et que, pour tous les lieux qu'il mentionne, il emploie la forme biblique du nom, j'y vois comme un relent idéologique, directement politique. Il ne parle pas de la Batanée, à l'époque romaine, où le pays s'appelle Batanaia, mais du pays de Bashan, la forme biblique du nom, qui remonte au $\mathrm{II}^{\mathrm{e}}$ millénaire av. J.-C., ou au début du $\mathrm{I}^{\mathrm{er}}$, et qui ne couvre d'ailleurs pas exactement le même espace. Lorsqu'il parle de la ville de Canatha, il emploie la forme qu'on utilise dans la Bible, Qenat ${ }^{33}$. Au bout du compte, on sent un relent expansionniste, annexionniste, qui ressemble à ce que font d'autres collègues israéliens, qui suivent les armées israéliennes lorsqu'elles avancent, et qui, dans tout 
bâtiment non identifié retrouvé sur le Golan, reconnaissent à coup sûr une synagogue $e^{34}$.

Mais on trouve évidemment des exemples dans l'autre camp. Lorsque les Syriens, selon l'idéologie ba'athiste officielle, font de Philippe dit l'Arabe un Arabe, c'est une annexion sans intérêt et dont il est d'ailleurs difficile de montrer qu'elle est mal fondée ${ }^{35}$. Lorsque l'on fait de Zénobie, "reine de Palmyre», qui fut en réalité simplement reine ${ }^{36}$, un quasi leader d'une sorte de front de libération de la Syrie au temps de l'occupation romaine, et que le ministre de la défense de Syrie vous explique que l'époque coloniale romaine est l'une des périodes les plus sombres de l'histoire de la Syrie, alors que vous êtes face aux merveilles de Palmyre, vous écarquillez les yeux. Pourquoi une époque sombre? Parce qu'un officiel assimile l'époque romaine, qui est une époque de domination, d'occupation, c'est incontestable, au colonialisme, donc à quelque chose de mauvais, de pervers. Le général Moustapha Tlass, qui a patronné un colloque à Homs et à Palmyre où nous étions ${ }^{37}$, a prononcé un discours d'introduction en ce sens. Lorsque les savants étrangers présents ont enfin parlé de Zénobie et d'Odeinat, en disant que la première n'était pas le leader du front de libération national puisqu'elle s'était proclamée impératrice de Rome, je vous le rappelle, et qu'il est difficile de faire sécession lorsqu'on se proclame soi-même chef de l'État en question, certains collègues syriens nous ont regardés sans comprendre. Là, l'histoire est annexée de telle façon que l'on ne peut revenir en arrière.

Voici ce que dit le général Moustapha Tlass, qui a été ministre de la Défense d'Hafez el-Assad, jusqu'au début de la présidence de Bachar el-Assad, de 1970 à 2003 ou 2004 : «Zainab (c'est-à-dire Zénobie) avait pris en charge le mouvement de libération au nom de tout l'Orient. » D'où tire-t-il cela? Je ne le sais pas. «Ou, plus spécifiquement, de tous les Arabes. » Difficile de donner une définition des Arabes. "L'Orient en général aurait dû réagir à ce que ce rôle impliquait et aligner ses forces sur un front unique. » Là, c'est le stratège qui parle. "Mais il y avait un autre facteur qui ne pouvait être ignoré : c'est la crainte du colonialisme d'affronter un État nouvellement indépendant. Quand la guerre romano-palmyrénienne éclata, l'Orient était sous l'occupation des Romains depuis plusieurs siècles, durant lesquels les forces romaines pratiquèrent les formes les plus hideuses d'oppression. Ce qu'ils avaient fait à Pétra et dans d'autres cités l'indique suffisamment. " Je cherche encore ce que les Romains ont fait à Pétra et quelles ont été ces "formes hideuses d'oppression", sans avoir trouvé. «Palmyre, soudainement éclatait en plein jour comme une grande puissance. Il est vrai que Palmyre avait aidé les Romains à détruire les juifs et avait combattu avec eux dans les guerres romano-perses sous le commandement romain.» C'est ambigu pour Tlass, ce comportement. Parce que d'un côté détruire les juifs c'est bien, mais le faire avec les Romains signifie que l'on aide le colonialisme !

Tout cela pour montrer comment l'histoire ancienne peut être récupérée dans un contexte d'hyper-nationalisme et un climat de guerre. On fait allusion à des choses sans savoir de quoi il s'agit, on ne tient aucun compte des textes, mais on récupère. Le travail des historiens n'est, évidemment, pas du tout pris en compte. Mais ne croyons pas que nous sommes nous-mêmes dégagés de toute influence. Comme tout historien, je suis sous influence. J'essaie de m'en tenir à une documentation et de donner l'explication qui me parait la plus conforme en fonction des critères scientifiques en usage dans ma communauté et sous le regard de mes collègues, pour aboutir à 
quelque chose qui n'est sûrement pas la vérité, mais qui essaie de s'en approcher le plus possible ou, plus exactement, bâtir un discours qui permette de comprendre au mieux le plus grand nombre des faits établis en notre possession. Adopter, en somme, une démarche différente de celle de Tlass, ou de celle de Paltiel et de son livre sur les princes clients, auquel je faisais précédemment allusion. Ne pas partir d'une idéologie à laquelle on agrège des faits et événements disparates, voire déformés, pour mettre l'histoire au service de son idéologie.

NATHAËL RECOURSÉ : Quelle diffusion une récupération idéologiquement orientée comme celle que vous avez décrite connaît-elle dans les sociétés du Proche-Orient contemporain? Peut-on en mesurer la portée, l'influence ? Y a-t-il, de la part des populations, une certaine perméabilité à ce discours?

Maurice Sartre : Si l'on observe l'évolution sur une quarantaine d'années, il y a des aspects négatifs et des aspects positifs. Le nationalisme, qui est pour moi une chose hideuse - je ne parle pas du patriotisme, je dis bien le nationalisme -, a eu pour effet de favoriser une certaine appropriation du patrimoine archéologique par la population. Palmyre était déjà, il y a quarante ans, un grand site, avec le Krak des chevaliers et un peu Saint-Siméon. Depuis, d'autres sont venus compléter la liste : Bosra, site inscrit au patrimoine de l'humanité, devenu l'un des sites les plus visités ; Apamée, qui était à l'abandon est devenu un site très visité ; etc. Il s'est développé un tourisme local, syrien, en Syrie. C'est l'aspect positif.

Malheureusement, la contrepartie espérée n'est pas sensible : à l'échelle locale, dans les villages, le patrimoine antique ne profite pas de cet engouement pour les antiquités. On se focalise sur quelques sites majeurs et on laisse tomber tout le reste, qui n'a pas été classé, en quelque sorte, comme patrimoine national. Néanmoins, localement se montent de petites associations culturelles de personnes intéressées par ce qu'il s'y passe. Ainsi, à Soueida, chez les druzes, qui sont très intéressés par l'histoire de leur région. Cela relève aussi d'un patriotisme local qui est particulièrement marqué ici.

Et puis, il y a des efforts, faibles et dispersés, mais réels. Par exemple, j’ai participé très activement à la réalisation d'un petit film d'animation pour sensibiliser les enfants du mohafazat de Soueida au fait qu'il existait des antiquités, c'est-à-dire des éléments de leur patrimoine, partout, dans chaque village. Le même travail a été fait pour Palmyre, où la sensibilisation est moins nécessaire. Ce film est projeté dans tous les villages, grâce à un bus qui circule d'école en école. C'est très modeste, mais c'est un début qu'il convenait d'encourager.

NATHAËL RECOURSÉ : Quel accueil reçoit, dans le Proche-Orient contemporain, la recherche, que celle-ci soit menée par des savants locaux ou par des savants étrangers, comme vous, comme d'autres? Quelle diffusion pour cette recherche? quels moyens? dans quelle mesure est-elle exposée (musées, publications, revues de vulgarisation)? quelles réalisations concrètes et quelles valorisations du savoir dans le cadre institutionnel? comment toutes ces choses s'articulent-elles?

Maurice Sartre : Il n'existe pas de réelle revue de vulgarisation, pas l'équivalent de L'Histoire, pour parler d'une revue que j'apprécie et qui joue, je crois, un rôle dans la valorisation de la recherche universitaire. Ceci signifie que le milieu savant est très largement coupé du grand public. Et, ce qui est fâcheux, c'est que nos collègues ne voient en général pas la nécessité d'entretenir des liens avec le grand public. Certes, 
c'est difficile : le niveau du grand public n'est pas celui d'un pays européen ; mais, si on ne commence pas un jour, on n'arrivera à rien.

Néanmoins cette curiosité existe. Je me souviens d'un colloque, en 1990, à Soueida, pour l'inauguration du musée de la ville, organisée par les autorités syriennes avec des savants français, parce que ce sont surtout eux qui ont travaillé là, et quelques collègues allemands. Dans une grande salle, avec au moins trois cents personnes, un public passionné suivait les débats et venait volontiers nous interroger durant les pauses. Il faut bien reconnaître que nous n'avions pas tous la même conception du dialogue avec le public et que certains ne répondaient qu'avec arrogance aux questions, certes naïves, d'un public plus chaleureux qu'instruit.

De ce côté, il y a encore des efforts à faire. Ma femme et moi avons toujours pris le temps, lorsque nous trouvons des inscriptions sur un terrain privé, d'expliquer au propriétaire le sens et le contenu des inscriptions découvertes lorsque, évidemment, nous les comprenons aussitôt. C'est un moyen de protéger l'antiquité, mais c'est aussi un devoir, un moyen de transmettre un petit élément de culture.

Il est de notre devoir d'apporter cet élément d'instruction; il y a donc ici un changement de mentalité à opérer. Pour le reste, je crois que l'avenir de la recherche passe nécessairement par la collaboration des uns et des autres. Globalement, les autorités scientifiques, en Syrie, en Jordanie ou au Liban, nous accueillent de façon extrêmement généreuse. Notre réponse doit être d'accueillir en retour le plus largement possible les étudiants locaux qui demain seront responsables de la recherche. Parce qu'il n'est pas normal que l'essentiel de la recherche archéologique en Syrie soit menée par des Français, des Américains, des Allemands, des Italiens, des Suisses, des Polonais, des Hongrois et que les Syriens soient presque absents des publications.

Il faut que cette collaboration encourage ces collègues à publier, ce qu'ils n'osent pas faire, en partie parce qu'ils ont peur de notre jugement. Il faut leur donner les moyens d'écrire, quitte à écrire avec eux. On sait bien qu'en Turquie ça a été la règle quasi générale pendant vingt ans. Maintenant, les collègues turcs écrivent seuls et ils écrivent très bien.

En même temps, ce qu'il faudrait, ce serait arriver à promouvoir plus systématiquement la traduction en arabe des ouvrages écrits dans d'autres langues. Or, malheureusement, la traduction en arabe pose d'énormes problèmes techniques, notamment celui des noms propres, qui sont systématiquement déformés; il faut faire le choix de garder les noms propres en français, en anglais ou dans une autre langue en caractères latins, faute de quoi le lecteur ne pourra jamais retrouver lesdits noms dans les grands dictionnaires occidentaux.

Il faudrait trouver un moyen pour d'avantage traduire en arabe. C'est un effort à faire de notre part; souvent les éditeurs sont prêts à céder les droits gratuitement ou pour un euro symbolique. Mais il faut évidemment qu'un effort parallèle soit fait de l'autre côté, et que cessent notamment les éditions pirates. Mon petit GallimardDécouvertes, La Syrie Antique ${ }^{38}$, a été publié par le ministère de la Culture à Damas, en arabe, sans aucune indication de provenance, et sans l'accord ni de l'éditeur, ni de l'auteur. On ne peut que se réjouir que nos travaux soient ainsi lisibles par un public arabophone, mais le respect du droit moral des auteurs n'est pas à négliger non plus. Ce type de pratique est un véritable frein, parce que les organismes comme le Centre 
national du livre, en France, qui aide volontiers à la traduction, refusent de traiter avec des pays qui ne respectent pas les règles internationales sur la propriété intellectuelle.

ADELINe GRAND-CLÉment : Vous avez dit que l'historien a le devoir d'être engagé dans le présent de sa cité, même quand il s'occupe, comme l'historien de l'antiquité que vous êtes, de champs d'étude éloignés à la fois dans le temps et dans l'espace. L'ouverture sur la société est nécessaire et salutaire pour montrer que l'étude des mondes anciens n'est pas repliée sur elle-même, et qu'elle ne consiste pas en l'étude d'un patrimoine qui serait idéalisé et figé à jamais; à l'inverse, elle peut contribuer à éclairer le temps présent. Quelqu'un comme Pierre Vidal-Naquet, dont vous avez suivi, je crois, l'enseignement, l'a magnifiquement montré ; il affectionnait particulièrement cette phrase de Marrou: "Le travail de l'historien n'est pas l'évocation d'un passé mort, mais une expérience vivante, dans laquelle l'historien engage l'expérience propre de sa destinée ${ }^{39}$. » On a eu l'occasion de rappeler vos multiples engagements en matière de diffusion du savoir : se sont-ils imposés à vous comme nécessaires, inhérents à votre métier, ou bien sont-ils nés de circonstances fortuites? Vous définiriez-vous comme un historien engagé ou comme un historien passionné, comme vous le disiez dans une belle phrase, «si l'historien n'a pas les yeux de l'amour, alors ça ne sert à rien »?

Maurice Sartre : Mes engagements ont été un peu le fruit du hasard, hasard qui est néanmoins rarement tout à fait fortuit ; je veux dire par là que si vous me demandez pourquoi j'ai été le premier président des «Rendez-vous de l'histoire », c'est parce qu'un jour Francis Chevrier a débarqué dans mon bureau en m'expliquant ce que Jack Lang voulait créer à Blois, en me disant qu'ils avaient constitué un comité scientifique, qu'ils cherchaient un président et qu'ils avaient pensé à moi. Cela ne relevait pas totalement du hasard. De même, lorsque Stéphane Khémis m'a demandé de rentrer au comité de rédaction de L'Histoire, ou que Philippe-Jean Catinchi m'a demandé d'écrire pour Le Monde des livres, ça ne devait pas être complètement le hasard : ils avaient des raisons précises de formuler cette demande. J'ai aussi été le président, jusqu'à octobre 2009, de l'Institut européen de l'histoire et des cultures de l'alimentation, ce qui a été aussi une autre aventure.

Dans le fond, la vraie raison de tout ceci, c'est que, d'une manière ou d'une autre, j'ai toujours aimé communiquer : c'est ma passion. Je ne le vis pas comme une passion violente, il ne faut pas exagérer; j'ai d'autres activités que d'écrire et faire de la recherche, tout de même : j'adore, par exemple, ne rien faire. Mais cela ne m'arrive pratiquement jamais. Tant pis! La vie est faite de contradictions. Ainsi, je déteste les voyages organisés. Mais vraiment, viscéralement! Et pourtant, lorsque des amis débarquent en Syrie, je ne peux pas m'empêcher de leur faire visiter la Syrie, parce que j'adore ça! J'ai envie de communiquer la passion que j'ai pour ce pays, passion qui peut être critique, certes, mais passion tout de même. Et bien je dirais que c'est un peu la même chose pour bien d'autres activités: je déteste administrer. Mais quand on m'a demandé d'être directeur de laboratoire, d'UFR, etc., j'ai toujours essayé de le faire du mieux possible.

Je crois qu'il y a aussi la passion de découvrir de nouvelles choses. Ce qui m'a poussé à accepter ce que l'on m'a proposé, parce que j'ai aussi fait de la radio, de la télévision, du cinéma..., c'est que j'ai beaucoup de peine à dire non à quelque chose que je n'ai jamais fait. J'ai failli faire un film avec Yannick Bellon, et puis, faute de financement, le projet ne s'est pas monté. C'est maintenant un peu tard, parce que je crois qu'un film est sorti sur le même sujet, Hypatie. Mais nous avions énormément travaillé sur le projet, j'ai passé des soirées entières avec Yannick Bellon pour essayer d'ajuster le 
scénario qu'elle avait élaboré. Nous discutions passionnément, je corrigeai des détails surprenants (par exemple les mosaïques au mur, comme dans les musées) mais j'apprenais beaucoup sur les contraintes d'un metteur en scène, l'importance des dialogues, etc.

Je crois donc que c'est le goût de la nouveauté qui m'a poussé à faire toutes ces expériences, un peu comme le goût des voyages. Là, par exemple, j'ai accepté d'aller à Nisibe (Nusaybin aujourd'hui, en Turquie), parce que je n'y étais jamais allé, alors que l'intervention m'a demandé un travail fou, car je n'avais rien à dire sur cette importante ville aux confins de Rome et de l'Empire perse. Plus profondément, si nous universitaires, nous chercheurs, nous ne faisons pas le travail de communication avec le grand public, on le laisse faire par d'autres qui risquent d'être peut-être plus habiles mais bien moins compétents scientifiquement et qui risquent de raconter n'importe quoi. Notre travail ne consiste pas en la seule érudition, il faut aller jusqu'au bout, et c'est pour cela que les "Rendez-vous de l'histoire ", la revue L'Histoire, Le Monde et tout le reste, m'ont semblé, à chaque sollicitation, des évidences.

Il y a tout de même des contreparties dont il faut se méfier: quand les médias commencent à connaitre un interlocuteur, ils sont persuadés qu'il n'y a que lui à interviewer quel que soit le sujet, non seulement dans le domaine de l'Antiquité, mais dans bien d'autres. Je dois pourtant en faire l'aveu, je ne sais pas tout, je ne suis pas compétent sur tout et je ne donnerai pas mon avis sur tout. Parce que si je suis un peu compétent pour l'histoire de l'Antiquité (et encore, pas sur tout), ça ne justifie pas que je donne un avis fondé sur le déménagement des archives en banlieue parisienne... Je ne suis pas du genre à signer des pétitions à tout va. Il m'est arrivé souvent de refuser: des gens de radio qui me téléphonaient pour me demander mon avis sur tel ou tel sujet. Je devais leur répondre que je n'avais pas forcément d'avis sur tout. D'un autre côté, écrire dans ces revues et journaux est aussi un formidable apprentissage. J'ai appris beaucoup à écrire en étant contraint : quand vous avez trois mille signes pour faire un compte rendu de livre, c'est très court. Mais cela veut aussi dire que, dès la première phrase, vous êtes obligé d'accrocher votre lecteur ; eh bien ! je peux vous assurer que, dans ces conditions, vous cherchez avec soin la première phrase!

C'est une excellente formation : je ne prétends pas être un bon écrivain, j'écris dans la douleur, mais l'on me reconnaît en général le mérite de la clarté. Je pense que je dois beaucoup à cette communication avec un public qui ne connaît rien ou qui est supposé ne rien connaître. Répondre à des questions naïves oblige aussi à réfléchir.

ADELINe GRAND-CLÉment : L'historien de l'Antiquité travaille sur des dossiers de sources fragmentaires, il a du mal à avoir des certitudes, il propose des hypothèses, et le grand public est, lui, plutôt avide de vérités, de certitudes. La transmission du savoir scientifique n'implique-t-elle pas un peu de dénaturer les choses, de les simplifier à l'excès ? Comment opérer la transmission en ne perdant pas la complexité des choses? Je pensais par exemple à la controverse autour du documentaire de Gérard Mordillat et Jérôme Prieur sur les premiers temps du christianisme, ou à la série Rome, qui a beaucoup de succès.

Maurice Sartre : À mon sens, les problèmes que posent l'écrit et l'image sont différents. À l'écrit, on simplifie toujours : lorsque vous faites le compte rendu d'un livre en trois mille ou en cinq mille signes, vous prenez des risques énormes et vous portez une appréciation sur un livre qui peut aller jusqu'à tuer le livre. Cela m'est 
arrivé quelques fois, pour des livres que je jugeais dangereux, pour diverses raisons scientifiques. Je pense, en effet, qu'il y a des livres dangereux parce que, sous l'apparence de la séduction, ils véhiculent des idées qui peuvent relever du délit, comme des idées racistes, par exemple - et le racisme ce n'est pas une opinion, c'est un délit. $\mathrm{Ou}$ alors parce qu'il relève du faux-semblant: je pense par exemple à un dictionnaire historique de la Gaule, paru il y a quelques années, qui était en fait un ramassis de citations d'auteurs du début du XIXe siècle, jamais mises entre guillemets, et seule une lecture attentive de la bibliographie permettait de comprendre le procédé malhonnête de rédaction de l'ouvrage ${ }^{40}$. Or, c'était un livre destiné aux étudiants, que l'on aurait retrouvé sur les rayons de toutes les bibliothèques, puisqu'il s'agissait d'une publication récente. Il y avait par exemple une entrée "Élagabale » (on se demande ce qu'il faisait dans un dictionnaire sur la Gaule), qui était un résumé de l'Histoire Auguste, qui disait plus ou moins, «Élagabale, empereur qui introduisit à Rome toutes les perversions de l'Orient». On voit quelles âneries pouvaient se retrouver dans toutes les copies, avec l'autorité d'un livre tout récent, en apparence !

Écrire est donc difficile. Dans un article de quelques pages, on est obligé de simplifier un peu, mais, pour ma part, j'essaie souvent de ne développer qu'une idée ou deux, au maximum. Je ne vais pas essayer de raconter le monde hellénistique en treize mille signes, c'est inutile. Je me limite donc à une seule idée, que je crois assez juste, assez proche de la réalité; je pense qu'à ce compte-là, on peut être à la fois clair, compréhensible et ne pas trahir ce que l'on veut dire.

Pour ce qui est des images, il faut en distinguer différents types. Avec les séries télévisées ou les films, comme Rome, comme Gladiator, on ne fait pas œuvre d'histoire. Lorsque j'écris dans L'Histoire, j'écris en tant qu'historien et, partant, je n'écris pas du roman. Dans la fiction, on a une liberté : lorsque j'ai travaillé à ce projet de film avec Yannick Bellon sur Hypatie, il y avait des aspects qui relevaient du roman. Par exemple, elle voulait la rajeunir parce qu'il fallait qu'elle soit séduisante; or moi je suis persuadé qu'au moment de sa mort, à Alexandrie en 415 - vous pouvez lire le dernier chapitre des Histoires grecques pour comprendre qui est Hypatie et comment se passent ses derniers instants ${ }^{41}$-, elle a au moins soixante-cinq ans. Dans ces conditions, il est plus difficile d'en faire non pas la maitresse mais au moins une amie de cœur du préfet d'Égypte que si elle en a trente-cinq.

Dans ces séries, les auteurs les plus sérieux ont, malgré tout, le respect de la vraisemblance historique. Comme historiens, nous n'avons qu'un squelette, un squelette qu'habille le romancier ou le cinéaste, à qui il met de la chair, de la peau, de la barbe... C'est pour cela que d'aucuns ont trouvé étrange que je défende le récit qu'avait fait Irène Frain de la vie de Cléopâtre. Elle ne l'a pourtant jamais intitulé « Histoire de Cléopâtre »; elle l'a appelé L'inimitable et désigné comme un « récit ». Je ne dis pas que tout ce qui est dedans est juste ; je ne le sais pas puisqu'il y a une foule de détails qui sont impossibles à connaître. Mais ce qui m'avait séduit dans ce livre, c'était que toute la partie documentée, c'est-à-dire ce que l'on connaît réellement du personnage, était bien intégrée, qu'il n'y avait pas de faute grave aux yeux de l'historien, de contresens historique ; ensuite, simplement, le talent de la romancière mettait la chair que nous, historiens, ne pouvons inventer.

D'une certaine manière, lorsque Pierre Briant écrit sa Lettre ouverte à Alexandre le Grand ${ }^{42}$, il procède un peu de la même manière. Il dit à Alexandre le Grand tout ce que 
sont ses certitudes d'historien, qui donnent chair au personnage, mais que l'historien n'a rien pour étayer, ne peut prouver, parce qu'on ne restituera jamais le vrai Alexandre et ses pensées.

C'est la même chose avec le livre, tout à fait merveilleux, de John Paul Meier sur Jésus, en quatre volumes - on attend le cinquième. Il mène une enquête qui aboutit à la conclusion qu'on ne pourra jamais connaître totalement le Jésus historique. On peut cerner le Jésus tel que les historiens essaient de le reconstituer, c'est-à-dire pas grand-chose au total.

Vous avez aussi fait allusion à autre chose, qui nous a entraînés dans des polémiques, voire des dénonciations, qui est la série de Gérard Mordillat et Jérôme Prieur. Cette série est extrêmement séduisante par bien des aspects et, notamment la première série, Corpus Christi, qui avait donné lieu à un livre intitulé Jésus contre Jésus ${ }^{43}$. J'avais dit tout le bien que j'en pensais. Mais, avec le temps, d'un épisode à l'autre, l'intérêt a faibli et les méthodes se sont gauchies. Le travers qu'a dénoncé Jean-Marie Salamito me paraît tout à fait exact ${ }^{44}$ : le procédé du montage qui consiste à juxtaposer des interviews de personnes qui n'ont jamais pu discuter entre elles, de faire répondre les intervenants à des questions dont le spectateur ne connaît pas la teneur, est, à la longue, un procédé dangereux pour l'historien. Je ne dis pas que c'est malhonnête, je ne l'ai, d'ailleurs, jamais écrit, mais ça peut être extrêmement dangereux. Jean-Marie Salamito avait parfaitement raison de montrer qu'en plus, la phrase récurrente de Loisy répétée à plusieurs reprises est le fils rouge qui permet de comprendre l'objectif principal de Gérard Mordillat - ce n'est pas tellement Jérôme Prieur qui est en cause dans l'affaire -, à savoir que l'Église a trahi Jésus ; ce qui est absurde parce que Jésus, sans l'Église, n'existerait pas. On est là dans un tout autre genre que les ouvrages évoqués plus haut, et plus loin encore des séries ou films historiques car la série tente de donner un sens à un événement capital, la naissance du christianisme, vise à la démonstration. Déceler des erreurs de méthode, voire des procédés de déformation, est donc non seulement légitime, mais nécessaire. Rendre compte d'un livre bien argumenté sur ce point n'est que se faire l'écho d'une polémique dont l'enjeu est essentiel. Livrer un travail au public, c'est naturellement s'exposer à la critique et lorsque celle-ci est honnête, c'est-à-dire qu'elle ne déforme pas votre travail luimême, elle doit être acceptée comme telle.

D'où ma stupeur devant la réaction violente et outrancière de Gérard Mordillat qui m'a envoyé, après la publication de mon compte rendu de Salamito, une lettre stupéfiante que j'ai évidemment conservée : on aurait été en URSS avant 1956, j'étais bon pour la Sibérie! Le vocabulaire de la lettre est exactement celui des procès staliniens; dès l'ouverture, j'y suis traité de "valet», non pas de l'impérialisme américain, mais de Salamito! Pourquoi regrettais-je en commençant ce long entretien que l'idéologie n'irriguait plus les débats historiques? Il y a au moins Gérard Mordillat pour maintenir la tradition!

PASCAL PAYEN : Nous avons eu un écho de ces débats, quand nous avons reçu Jean-Marie Salamito qui nous a raconté comment il avait été amené, alors que ce n'est pas du tout sa manière d'être en tant qu'historien, d'une certaine façon par nécessité, intellectuelle et scientifique, à composer un pamphlet pour préciser ces choses.

Maurice Sartre: Oui, je lui avais fait suivre une copie de la lettre de Gérard Mordillat. Il avait, pour sa part, pris position parce que Le Monde de la Bible lui avait demandé un avis. Il avait émis des réserves et Mordillat est quelqu'un qui n'accepte 
pas la moindre réserve. La pétition des collègues dans Libération, dont il y a tout lieu de croire qu'elle a été rédigée par Gérard Mordillat lui-même, disait à la fin que non seulement l'œuvre était irremplaçable, mais qu'elle devrait être obligatoire dans toutes les écoles! Décidément, il y a de la nostalgie de l'époque du petit père des peuples!

ADELINE GRAND-CLÉMENT : À un moment où les sciences humaines et sociales en général et les sciences de l'Antiquité en particulier semblent connaître une crise de légitimité au sein de la société, j'avais envie de vous demander, un peu naïvement, à quoi sert l'histoire ancienne dans la société d'aujourd'hui. En quoi les « Anciens » sont-ils « modernes »?

Maurice Sartre : Je crois que c'est une question fondamentale. L'histoire en général, disons-le d'emblée, ça ne sert à rien ; mais essayons sans, et nous verrons bien. La crise actuelle ne fait que souligner le caractère indispensable des sciences humaines. Je ne fais pas de place particulière à l'histoire ancienne, que je considère comme l'une des branches de la discipline historique. Notre discipline reste un élément indispensable de la formation des individus et, notamment, de la formation des citoyens. Or, dans les pays où l'histoire n'existe pas ou est entièrement soumise à l'idéologie, il n'y a pas non plus de citoyens. Il y a des sujets, peut-être, d'un régime quelconque. L'histoire, même lorsque l'on fait un travail éloigné de toute préoccupation contemporaine, apprend à réfléchir, à coordonner les informations : c'est-à-dire, au bout du compte, à ne pas gober n'importe quoi ! L'histoire est un moyen de développer l'esprit critique, même lorsque l'on travaille sur des sujets dégagés - en apparence - de toute relation directe avec le monde contemporain. Grâce à l'histoire, on peut ouvrir un grand quotidien du soir et l'appréhender de façon critique.

Ce n'est pas un hasard si les œuvres historiques fondamentales ne se développent que dans les États démocratiques. Existe-t-il une œuvre majeure de l'historiographie contemporaine qui sorte d'une dictature? Je n'en connais pas. En retour, la démocratie a besoin des sciences humaines. Les hommes politiques éphémères qui ne pensent qu'au profit, aux lois du marché et ne voient pas plus loin que leur horizon personnel au point de penser et d'oser dire que la lecture d'un roman du XvII siècle n'a plus aucun intérêt, ces gens-là sont des guignols qui relèvent de l'épiphénomène, même s'ils nous font perdre un temps précieux puisqu'il faut ensuite corriger les effets de leurs mauvais coups.

Ce qui compte, c'est le fond de la société. Je suis très frappé de voir que les éditeurs en France font leurs meilleures ventes avec les livres de sciences humaines. Ils sont très demandeurs d'histoire! Quand on voit un éditeur comme Fayard accepter de publier un ouvrage de mille deux cents pages sur la Syrie antique ${ }^{45}$ ou un ouvrage de mille quatre cents pages sur l'empire perse, comme il l'a fait avec Pierre Briant ${ }^{46}$, sans demander de supprimer une ligne ou un mot de grec, on a envie de dire « chapeau !». Il existe des éditeurs pour qui les sciences humaines, même les plus " anecdotiques ", sont indispensables, outre qu'elles leur permettent de gagner de l'argent. Ce qui est assez moral ; ça m'embêterait que mes éditeurs perdent de l'argent, tout de même !

CORINNE BONNET : II me semble que nous avons amplement balayé à la fois les intérêts de Maurice Sartre et toutes les questions d'actualité, y compris le rapport entre présent et 
passé sous toutes ses formes, de la recherche la plus pointue à l'ouverture sur la société. Un grand merci à notre hôte pour sa franchise, pour l'absence de langue de bois.

Maurice Sartre : C'est moi qui voudrais vous remercier vraiment du fond du cœur pour cette journée, parce que ça a été un grand plaisir de vous revoir tous, de rencontrer de jeunes et moins jeunes chercheurs, d'avoir eu aussi un public si nombreux et merci aussi de m'avoir donné la possibilité de réfléchir à ce que je fais, démarche que je crois salutaire. Je n'ai pas de doute sur ma passion. Il reste cependant une question que vous ne m'avez pas posée, mais à laquelle je vais répondre quand même: pourquoi ai-je choisi de travailler sur la Syrie? J'y ai beaucoup réfléchi et je crois que ça a tenu à la magie d'un mot. Lorsque j'ai été jeune agrégé, avant même d'avoir un poste dans le second degré, j'ai été recruté comme assistant à l'université de Clermont-Ferrand par Claude Mossé. Lorsque je suis rentré en France (j'ai été recruté in absentia, étant alors en fouilles en Afghanistan), je devais trouver un sujet de thèse. Je suis allé voir Jean Pouilloux, à Lyon, et il m'a expliqué qu'il avait reçu les archives d'un savant allemand qui avait émigré aux États-Unis, qui s'appelait Brünnow, mort à Princeton en 1917, et qui avait commencé le corpus des inscriptions grecques de l'Arabie. Ne me demandez pas ce qu'il a dit après : une fois prononcé le mot "Arabie ", j'ai vu des étoiles partout et j'étais déjà sur un tapis volant en route vers l'Arabie. Le lendemain, j'ai cherché où pouvait être cette Arabie où il y avait des inscriptions grecques : je me suis alors aperçu que c'était la Syrie du Sud, la Jordanie et le nord de l'Arabie saoudite. Donc ce n'était pas du tout la péninsule arabique comme je l'avais cru dans ma grande naïveté. Tout ça pour dire que la magie du mot a opéré et que mon orientation s'est décidée là-dessus, non à la suite d'une analyse raisonnée et raisonnable de ce qu'il convenait d'étudier. Pour moi, l'Arabie, quand j'étais enfant et adolescent, c'était l'endroit où jamais je ne pourrais aller, parce que c'était un pays interdit, fermé aux étrangers, donc fascinant. On l'a oublié aujourd'hui où cinq millions de touristes se pressent à Dubaï chaque année ; dans les années 1960, c'était un monde fermé, impossible d'accès. Une vraie passion naît plus sûrement d'une image que de la raison. J'ai au moins acquis cette certitude.

\section{NOTES}

1. M. SARTRE, Inscriptions grecques et latines de la Syrie, $13: 1$, Bostra (Bibliothèque archéologique et historique de l'Institut français d'archéologie du Proche-Orient), Paris, Geuthner, 1983.

2. M. SARTRE, Bostra : des origines à l'Islam, Paris, Geuthner, 1985.

3. M. SARTRE, L'Orient romain : provinces et sociétés provinciales en Méditerranée orientale d'Auguste aux Sévères (31 av. J.-C. - 235 ap. J.-C.), Paris, Seuil, 1991.

4. M. SARTRE, D'Alexandre à Zénobie. Histoire du Levant antique, IV siècle avant J.-C. - III siècle après J.-C., Paris, Fayard, 2001, 2e éd. 2003.

5. M. SARTRE, L'Asie Mineure et l'Anatolie, d'Alexandre à Dioclétien, IV siècle av. J.-C. - III siècle ap. J.-C., Paris, Armand Colin, 1995.

6. M. SARTRE, L'Anatolie hellénistique de l'Egée au Caucase, Paris, Armand Colin, 2003. 
7. M. SARTRE, Histoires grecques, Paris, Seuil, 2006.

8. M. SARTRE, La Syrie antique, Paris, Gallimard (Découvertes, 426), 2002.

9. P. VEYNE, Le pain et le cirque. Sociologie historique d'un pluralisme politique, Paris, Seuil, 1976.

10. M. SARTRE, « Une histoire de sigma brisé ou l'impérialisme athénien », Histoires grecques, Paris, Seuil, 2006, p. 137-143.

11. Promesse qu'il n'a heureusement pas tenue, puisqu'il publie en octobre 2010 un livre sur la peinture italienne.

12. Je dois revenir sur cette affirmation car, entre le moment de ce séminaire et le moment où je le relis, ont paru le Périclès de Vincent Azoulay (Armand Colin, 2010), l'étude de Pauline schmitt PANTEL sur la fabrique des Hommes Illustres (Aubier, 2009), quelques thèses novatrices (Julien Fournier, Aude Cassayre). Il faut donc juger sur la plus longue durée et ne pas désespérer.

13. G. BONGARD-LEVINE, C. BONNET, Y. LITVINENKo et A. MARCONE, Mongolus Syrio salutem optimam dat. La correspondance entre Mikhail Rostovtzeff et Franz Cumont, Mémoires de l'Académie des Inscriptions et Belles-Lettres, Paris, De Boccard, 2007.

14. M.I. RostovtZefF, The Social and Economic History of the Hellenistic World, Oxford, Clarendon, 1941.

15. M.I. RostovtZEFF, The Social and Economic History of the Roman Empire, Oxford, Clarendon, 1926 ; The Social and Economic History of the Hellenistic World, Oxford, 1941.

16. Il s'agit de la première des deux études, celle sur l'Empire romain.

17. S. SAND, Comment le peuple juif fut inventé. De la Bible au sionisme, Paris, Fayard, 2008.

18. “A-t-on inventé le peuple juif ?”, Le Débat, 158, janvier-février 2010, p. 177-184, au sein d'un large débat très contradictoire.

19. M. SARTRE, "De Pétra à Jérusalem... et retour !", in East and West. Papers in Ancient History presented to Glen W. Bowersock, Harvard, Harvard University Press, 2008, p. 159-180.

20. P. VEYNE, introduction à G. DEGEORGE, Palmyre, métropole caravanière, Paris, 2001.

21. Il est utilisé pour purifier l'haleine.

22. M. SARTRE, "Uriner devant Aphrodite ou Juifs et Grecs six siècles plus tard", Histoires grecques, p. 417-425.

23. Cf. C. ORRIEUX - E. WILL, Ioudaïsmos - Hellènismos - Essai sur le Judaïsme judéen à l'époque hellénistique, Nancy, Presses Universitaires de Nancy, 1986.

24. Code Théodosien, XIII, 3, 5 = Code de Justinien, X, 53, 7 .

25. Syria, 64, 1987, p. 168 : M. SARTRE, compte rendu de P. Leriche, Fouilles d'Aï-Khanoum. V. Les remparts et les mouvements associés, Paris, De Boccard, 1986.

26. H. BRU - F. KIRBIHLER - S. LEBRETON, L'Asie mineure dans l'antiquité: échanges, populations et territoires: regards actuels sur une péninsule. Actes du colloque de Tours, 21-22 octobre 2005, Rennes, PUR, 2009.

27. H.-P. FRANCFORT (éd.), Nomades et sédentaires en Asie centrale: apports de l'archéologie et de l'ethnologie, Paris, Éditions du CNRs, 1990.

28. http://www.ifporient.org/, site consulté les 11, 12, 13 mai 2010.

29. P. Clauss BALty (ss la dir.), Hauran III. L'habitat dans les campagnes de Syrie du Sud aux époques classique et médiévale (BAH 208), Beyrouth, IFPO, 2008.

30. C. POUDERON - M. SARTRE, «Un marchand d'épigrammes à Maaga de Batanée (Syrie) », ZPE 160 (2007), p. 51-58.

31. B. CROCE, Théorie et Histoire de l'historiographie, Genève, Droz, 1968, p. 14.

32. Y. LAcostE, La géographie, ça sert, d'abord, à faire la guerre, Paris, Maspéro, 1976 (rééd. La Découverte, 1985).

33. E. PALTIEL, Vassals and rebels in the Roman Empire: Julio-Claudian policies in Judaea and the kingdoms of the East, Bruxelles, 1991.

34. D. URMAN, The Golan : a profile of a region during the Roman and Byzantine periods, Oxford, 1985. 
35. En résumé, il est probable que Philippe est le descendant de colons romains venus peut-être de la côte phénicienne (Bérytos ?) et son épithète d'Arabe est seulement liée au fait qu'il est né dans la province d'Arabie. Ajoutons que pour les bons Romains de souche, ce n'était sûrement pas valorisant.

36. Elle porte le titre de reine parce qu'elle est l'épouse d'Odeinat, « roi des rois », titre qu'il a pris après avoir battu Shapur $\mathrm{I}^{\mathrm{er}}$, roi sassanide qui portait ce titre. Il n'est en rien « roi de Palmyre ", titre inconnu.

37. M. al-HAYEK, M. MAQDISI et M. ABDULKARIM, Zenobia and Palmyra. Proceedings of the International Conference, (Homs-Palmyre, 19-21 octobre 2002), Homs, 2002.

38. M. SARTRE, La Syrie antique, Paris, Gallimard (Découvertes 426), 2002.

39. Cité par P. Vidal-Naquet, Le choix de l'Histoire, Paris, Arléa, 2004, p. 19.

40. J.-P. Рісот, Dictionnaire historique de la Gaule : des origines à Clovis : d'après des documents originaux et des textes du XIX ${ }^{e}$ siècle et contemporains, Paris, La Différence, 2002.

41. M. SARTRE, «La mort d'Hypathie ou Rester païen dans un monde chrétien », Histoires grecques, p. 437-447.

42. P. BRIANT, Lettre ouverte à Alexandre le Grand, Arles, Actes Sud, 2008.

43. G. MORDILLAT - J. PRIEUR, Jésus contre Jésus, Paris, Seuil, 2005.

44. J.-M. SALAmito, Les chevaliers de l'Apocalypse. Réponse à MM. Mordillat et Prieur, Paris, Lethielleux, 2009.

45. M. SARTRE, D'Alexandre à Zénobie. Histoire du Levant antique, IV siècle avant J.-C. - III $I^{e}$ siècle après J.C., Paris, Fayard, 2001, 2e éd. 2003.

46. P. BRIANT, Histoire de l'empire perse, Paris, Fayard, 1996.

\section{AUTEUR}

\section{MAURICE SARTRE}

Professeur émérite à l'Université François-Rabelais, Tours Membre senior de l'Institut Universitaire de France Maison de l'Orient et de la Méditerranée-Jean Pouilloux, Lyon sartre@wanadoo.fr 